TRANSACTIONS OF THE

AMERICAN MATHEMATICAL SOCIETY

Volume 351, Number 12, Pages 5051-5088

S 0002-9947(99)02231-X

Article electronically published on August 25, 1999

\title{
ON THE NORMAL SUBGROUPS OF $G_{2}(A)$
}

\author{
DOUGLAS L. COSTA AND GORDON E. KELLER
}

\begin{abstract}
We give a characterization theorem for the $E(A)$-normalized subgroups of $G_{2}(A)$, where $A$ is any commutative ring. This is the last of the simple Chevalley-Demazure group-schemes for which such a theorem is lacking.
\end{abstract}

\section{INTRODUCTION}

In this paper we prove a theorem (3.6) which characterizes the $E(A)$-normalized subgroups of $G_{2}(A)$ for an arbitrary commutative ring $A$. The theorem brings to completion the larger project of finding characterization theorems for $E(A)$ normalized subgroups of simple Chevalley-Demazure group-schemes $G(A)$ of rank $\geq$ 2 over arbitrary commutative rings. The bulk of the project was done by Vaserstein in his essential paper [16]. There he proved the standard characterization theorem, given by a "ladder" corresponding to an ideal, for all commutative rings for all $G$ of rank $\geq 2$ except those of types $B_{2}=C_{2}$ and $G_{2}$. Vaserstein also suggested in that paper that previous work by Abe [1] and Abe-Suzuki [3] could be improved to yield more. This was accomplished by Abe in [2], wherein he showed that $E(A)$ normalized subgroups of $G(A)$ could be characterized by ladders determined by "admissible pairs" of ideals, with the exception that for $G$ of type $B_{2}=C_{2}$ or $G_{2}$ rings $A$ having residue class fields of two elements must be excluded. Our paper [8] subsequently succeeded in removing this restriction for $G$ of type $C_{2}$ (and so also for $G$ of type $B_{2}$ ) by recognizing that the presence of residue fields of two elements required lowering the bottom rung of the ladder. This left only the case $G$ of type $G_{2}$ to be finished; the present paper does so by applying the "lower bottom rung" idea of [8] (see also [7]) to this case.

We now give a more explicit description of our results. Let $A$ be an arbitrary commutative ring and denote by $G_{2}(A)$ the group of type $G_{2}$ defined over $A$. The main theorem (3.6) is that a subgroup $N$ of $G_{2}(A)$ is $E(A)$-normalized if and only if $C\left(J, J^{\prime}\right) \subseteq N \subseteq G\left(J, J^{\prime}\right)$ for a pair of ideals $J, J^{\prime}$ satisfying the condition $J_{3} \subseteq J^{\prime} \subseteq J$. Here, $J_{3}$ denotes the ideal of $A$ generated by all elements of the form $x^{3}$ and $3 x$ for all $x$ in $J$, so that $\left(J, J^{\prime}\right)$ is an admissible pair in the sense of Abe. The bracketing groups $C\left(J, J^{\prime}\right)$ and $G\left(J, J^{\prime}\right)$ are defined in Section 1 below. In essence, $C\left(J, J^{\prime}\right)$ is the mixed commutator group $\left[E(A), E\left(J, J^{\prime}\right)\right]$, where $E\left(J, J^{\prime}\right)$ is the $E(A)$-normalized subgroup generated by all elements $x_{\alpha}(j)$ with $\alpha$ long and

Received by the editors April 1, 1997 and, in revised form, May 22, 1997.

1991 Mathematics Subject Classification. Primary 20H05; Secondary 20 G35.

Research partially supported by NSA grant MDA 904-94-H-2008 and NSF grant DMS-9622899.

(C)1999 American Mathematical Society 
$j \in J$ and all $x_{\beta}\left(j^{\prime}\right)$ with $\beta$ short and $j^{\prime} \in J^{\prime}$. [In [2], Abe's lower bracketing group is $E\left(J, J^{\prime}\right)$, which is too large for the two-element residue field case.] The group $G\left(J, J^{\prime}\right)$ is a congruence group: it consists of all elements whose level is contained in $J$ and whose " $A_{2}$-level" is contained in $J^{\prime}$. This is proved in (3.7). [There is a naturally embedded copy of $S L(3, A)$ in $G_{2}(A)$ and the $A_{2}$-level of $N$ is the level ideal of the intersection $N \cap S L(3, A)$; see Definition (1.8).] In (3.10) we show that $G\left(J, J^{\prime}\right)$ and $C\left(J, J^{\prime}\right)$ are, in fact, normal in $G_{2}(A)$. Section 3 also shows that there is a perfect correspondence between admissible pairs $\left(J, J^{\prime}\right)$ and the groups $G\left(J, J^{\prime}\right)$.

There are some other salient features of the paper which deserve mention here.

First, a key to our approach is to use the well-known fact that $G_{2}(A)$ can be thought of as the automorphism group of the split octonion algebra, $\mathcal{O}(A)$, over $A$. Taking this point of view allows us to realize $G_{2}(A)$ as a group of $8 \times 8$ matrices in which we may carry out many of our computations. This representation of $G_{2}(A)$ is developed in an appendix and leads to a new matrix characterization of $G_{2}(A)$ in Section 2 (Theorem (2.1)).

Second, in the spirit of [9], we give a homomorphism on $E\left(J, J^{\prime}\right)$ whose kernel is precisely $C\left(J, J^{\prime}\right)$. The homomorphism is explicit in the sense that it is given by a polynomial in the entries of the matrices constituting elements of $G_{2}(A)$. It thus provides an effective way to decide which elements of $E\left(J, J^{\prime}\right)$ are in $C\left(J, J^{\prime}\right)$. This is done in Theorem (1.18).

Finally, Section 4 provides a computational method for determining the " $A_{2}$ level" of a matrix in $G_{2}(A)$. This means in principle that for a given $E(A)$ normalized subgroup $N$, one can explicitly determine the admissible pair $\left(J, J^{\prime}\right)$.

The problem of characterizing normal subgroups of linear and classical groups has a history dating back over a century. Initially, of course, the primary focus was on groups defined over fields. Later efforts produced theorems for groups defined over special classes of rings, such as algebraic number rings satisfying certain conditions. Much of the recent work on the characterization problem was inspired by the work of Bass-Milnor-Serre [5] on the congruence subgroup problem and, to some extent, by the techniques developed by Suslin and Vaserstein in their attacks on the Serre conjecture. The surprising result of Wilson [18], characterizing $E(n, A)$-normalized subgroups of $G L(n, A)$ for all $n \geq 4$ and all commutative rings $A$, showed everyone that theorems for arbitrary commutative rings were possible. This provided the impetus leading to Golubchik [10], [11], Vaserstein [15], and Borevich-Vavilov [6], in each of which Wilson's theorem was extended to $n \geq 3$.

The extension of the latter work to groups of rank $\geq 2$ is principally due to Vaserstein, Abe, and Taddei. Taddei [14] proved the normality of $E(A)$ in $G(A)$. Vaserstein [16] used Taddei's result to prove that for any ideal $J$ of $A,(1) E(J)$ is normal in $G(A)$, and $(2)[E(A), G(J)] \subseteq E(J)$. [See definitions below. These theorems are crucial in our proofs.] This enabled Vaserstein [16] and Abe [2] to prove the omnibus theorems described above, and gave us the tools to complete the characterization theorems for groups of type $C_{2}$ in [8] and groups of type $G_{2}$ in the present paper. For more detailed background and history we refer the reader to the excellent survey [17] by Vavilov, the introduction to [16], and the comprehensive text of Hahn and O'Meara [12].

We are indebted to John Faulkner and Bruce Allison for their gracious and generous advice and counsel throughout this work. 


\section{The group $G_{2}(A)$ : Basic Results}

Let $A$ be a commutative ring with identity. Let $G_{2}$ be the almost simple Chevalley-Demazure group scheme with root system $\Phi$, where $\Phi$ is as in Figure 1. The group $G_{2}(A)$ is the group of $A$-valued points of $G_{2}$. As discussed in the appendix, we may simultaneously view $G_{2}(A)$ as the group of $A$-automorphisms of the split Cayley algebra over $A, \mathcal{O}(A)$.

We now set up some notational conventions. Let $E(A)$ be the subgroup of $G_{2}(A)$ generated by all elementary unipotent elements $x_{\phi}(t)$ with $\phi \in \Phi$ and $t \in A$. For any ideal $J$ of $A$, let $G(A ; J)=G(J)$ be the group of all elements of $G_{2}(A)$ congruent to the identity modulo $J$, i.e., the principal congruence subgroup defined by $J$. By (A.12), $G(J)$ is also the inverse image of the (trivial) center of $G_{2}(A / J)$ under the canonical homomorphism $G_{2}(A) \rightarrow G_{2}(A / J)$.

If $X \in G_{2}(A)$, the level ideal of $X, \ell(X)$, is defined to be the smallest ideal $J$ such that $X$ is a scalar modulo $J$. By (A.12), $\ell(X)$ is the ideal generated by the entries of $X-I$; moreover, $\ell(X)$ is the smallest ideal $J$ such that $X \in G(J)$. For a subgroup $H$ of $G_{2}(A)$, we set $\ell(H)=\sum_{X \in H} \ell(X)$. Clearly, $\ell(H)$ is the smallest ideal $J$ such that $H \subseteq G(J)$.

For a subset $S$ of $A$ we denote by $E(A ; S)$ or $E(S)$ the normal subgroup of $E(A)$ generated by the elements $x_{\phi}(t)$ for all $\phi \in \Phi$ and all $t \in S$.

Let $\Phi_{s}$ be the set of short roots in $\Phi$, and let $\Phi_{\ell}$ be the set of long roots in $\Phi$. If $S, S^{\prime}$ are two subsets of $A$, we denote by $E\left(A ; S, S^{\prime}\right)$ or $E\left(S, S^{\prime}\right)$ the normal subgroup of $E(A)$ generated by

$$
\left\{x_{\phi}(s) \mid \phi \in \Phi_{s}, s \in S\right\} \cup\left\{x_{\phi}\left(s^{\prime}\right) \mid \phi \in \Phi_{\ell}, s^{\prime} \in S^{\prime}\right\} .
$$

Note that $E(S)=E(S, S)$. With these conventions we may also define certain "generic" subgroups we will need: For ideals $J, J^{\prime}$, set $G\left(J, J^{\prime}\right)=E\left(J, J^{\prime}\right) \cdot G\left(J^{\prime}\right)$.

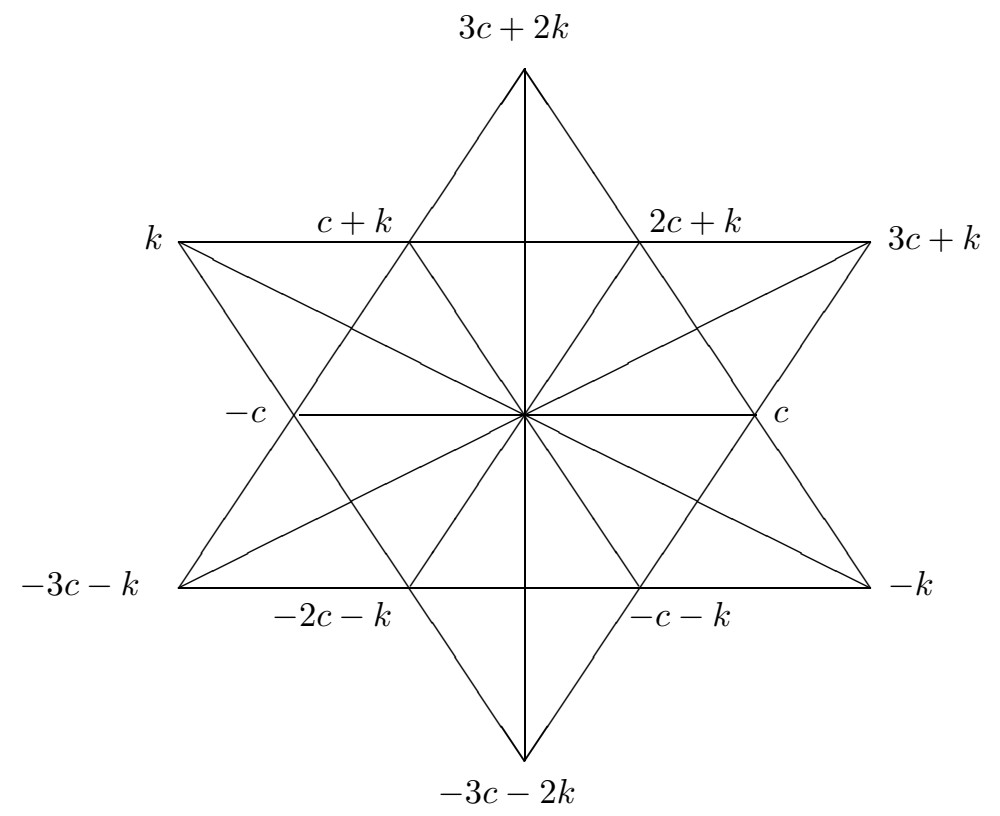

Figure 1 
For $\phi \in \Phi$ and $t \in A$, set $z_{\phi}(t)=x_{-\phi}(t) x_{\phi}(t)$. Then for any subset $S$ of $A$, we set $C(S)$ equal to the normal subgroup of $E(A)$ generated by $\left\{z_{\phi}(t) \mid \phi \in \Phi, t \in S\right\}$; and for two subsets $S, S^{\prime}$ of $A$ we let $C\left(S, S^{\prime}\right)$ be the normal subgroup of $E(A)$ generated by

$$
\left\{z_{\phi}(t) \mid \phi \in \Phi_{s}, t \in S\right\} \cup\left\{z_{\phi}(t) \mid \phi \in \Phi_{\ell}, t \in S^{\prime}\right\} .
$$

For an ideal $J$ of $A$ and a positive integer $n$, we define $J_{n}$ to be the ideal of $A$ generated by $\left\{t^{n} \mid t \in J\right\} \cup\{n t \mid t \in J\}$. We shall be particularly concerned with $J_{3}=\left(t^{3}, 3 t \mid t \in J\right)$. Another ideal we will need is

$$
v n_{2}(J)=\left(\left\{a^{2}-a \mid a \in J\right\}\right)=v n_{2}(A) \cap J
$$

$($ See $[7,9]$.

Embeddings of $S L(2, A)$ and $S L(3, A)$ in $G_{2}(A)$. In this section we view $G_{2}(A)$ as $\operatorname{Aut}(\mathcal{O}(A))$. Observing, as in the appendix, that the subalgebra $\mathcal{E}$ of $\mathcal{O}(A)$ spanned by $u_{1}=e_{11},-u_{2}=e_{12}, u_{6}=e_{21}$, and $u_{5}=e_{22}$ is a $2 \times 2$ matrix algebra over $A$, we note that $f=f_{12}+f_{21}$ is a unit in $\mathcal{O}(A)$ and that $\mathcal{O}(A)=\mathcal{E}+f \mathcal{E}$ is a direct sum decomposition of $\mathcal{O}(A)$. For an element $Y$ of $S L(2, A)$, this allows us to define an automorphism $\sigma(Y) \in G_{2}(A)$ by the rule: $\sigma(Y): \mathbf{e}+f \mathbf{e}^{\prime} \mapsto$ $Y^{-1} \mathbf{e} Y+Y\left(f \mathbf{e}^{\prime}\right)$, for any $\mathbf{e}, \mathbf{e}^{\prime} \in \mathcal{E}$, where $\mathbf{e}, \mathbf{e}^{\prime}$ are viewed as $2 \times 2$ matrices. Then $\sigma: S L(2, A) \rightarrow G_{2}(A)$ is an embedding of $S L(2, A)$ in $G_{2}(A)$. There are three such embeddings. We shall sometimes write " $S L(2, A)$ " to denote one of these subgroups of $G_{2}(A)$.

Note that with respect to the representation chosen for $G_{2}(A)$ in the appendix, if $Y=\left[\begin{array}{ll}\alpha & \beta \\ \gamma & \delta\end{array}\right] \in S L(2, A)$, then

$$
\sigma(Y)=\left[\begin{array}{cccccccc}
\delta \alpha & -\delta \beta & 0 & 0 & -\gamma \beta & -\gamma \alpha & 0 & 0 \\
-\gamma \delta & \delta^{2} & 0 & 0 & \gamma \delta & \gamma^{2} & 0 & 0 \\
0 & 0 & \alpha & 0 & 0 & 0 & 0 & -\beta \\
0 & 0 & 0 & \alpha & 0 & 0 & \beta & 0 \\
-\beta \gamma & \beta \delta & 0 & 0 & \alpha \delta & \alpha \gamma & 0 & 0 \\
-\beta \alpha & \beta^{2} & 0 & 0 & \alpha \beta & \alpha^{2} & 0 & 0 \\
0 & 0 & 0 & \gamma & 0 & 0 & \delta & 0 \\
0 & 0 & -\gamma & 0 & 0 & 0 & 0 & \delta
\end{array}\right]
$$

Next, suppose that $Y \in S L(3, A)$. Using the notation $\left(\begin{array}{cc}\rho & v \\ w & \sigma\end{array}\right)$ for an element of $\mathcal{O}(A)$ (see appendix), we define $\tau(Y) \in G_{2}(A)$ by $\tau(Y):\left(\begin{array}{cc}\rho & v \\ w & \sigma\end{array}\right) \mapsto\left(\begin{array}{cc}\rho & Y^{T} v \\ Y^{-1} w & \sigma\end{array}\right)$. Then $\tau$ is an isomorphism of $S L(3, A)$ into $G_{2}(A)$. We denote the image of $\tau$ by $S L(3, A)$. Using the matrix representation of $G_{2}(A)$ in the appendix,

$$
\tau(Y)=\left[\begin{array}{ll}
a & b \\
c & d
\end{array}\right]
$$

where

$$
a=\left[\begin{array}{cccc}
1 & 0 & 0 & 0 \\
0 & & & \\
0 & & Y^{T} & \\
0 & & &
\end{array}\right], \quad d=\left[\begin{array}{cccc}
1 & 0 & 0 & 0 \\
0 & & & \\
0 & & Y^{-1} & \\
0 & & &
\end{array}\right], \quad b=c=0
$$


Conversely, any $X$ in $G_{2}(A)$ with $b(X)=c(X)=0$ and

$$
a(X)=a=\left[\begin{array}{cccc}
1 & 0 & 0 & 0 \\
0 & & & \\
0 & & M & \\
0 & & &
\end{array}\right]
$$

is in $S L(3, A)$. (See the appendix.)

Under these embeddings any $Y$ in $S L(2, A)$ or $S L(3, A)$ which is elementary is mapped to a matrix $X$ in $G_{2}(A)$ having upper left $4 \times 4$ block $a(X)$ elementary. By (A.8), the elementary matrix $a(X)$ determines $X$ completely, so we may specify these elements by specifying this block. It can be shown that the images of the elementary matrices in $S L(2, A)$ under the (three) maps $\sigma$ are the elementary unipotent elements $x_{\phi}(t), \phi \in \Phi_{s}$. The images of the elementary matrices in $S L(3, A)$ under the map $\tau$ are the $x_{\phi}(t), \phi \in \Phi_{\ell}$.

In this paper, we choose the root $k$ so that when $x_{k}(t)$ is written as an $8 \times 8$ matrix, its upper left block is

$$
a\left(x_{k}(t)\right)=\left[\begin{array}{llll}
1 & 0 & 0 & 0 \\
0 & 1 & t & 0 \\
0 & 0 & 1 & 0 \\
0 & 0 & 0 & 1
\end{array}\right]=I+t e_{23} .
$$

At this point there is some freedom of choice as to the representation of $x_{3 c+k}(t)$. One of the possible choices, which we now make, is to pick $x_{3 c+k}(t)$ so that

$$
a\left(x_{3 c+k}(t)\right)=I+t e_{34} .
$$

These two elections actually force the remaining root groups to correspond to particular one-parameter elementary subgroups (cf. (1.1)). One possible choice of these representations is as follows:

$$
\begin{aligned}
a\left(x_{3 c+2 k}(t)\right) & =I+t e_{24}, \\
a\left(x_{c}(t)\right) & =I-t e_{31}, \\
a\left(x_{c+k}(t)\right) & =I-t e_{21}, \\
a\left(x_{2 c+k}(t)\right) & =I+t e_{14}, \\
a\left(x_{-k}(t)\right) & =I+t e_{32}=a\left(x_{k}(t)\right)^{T}, \\
a\left(x_{-3 c-k}(t)\right) & =I+t e_{43}=a\left(x_{3 c+k}(t)\right)^{T}, \\
a\left(x_{-3 c-2 k}(t)\right) & =I+t e_{42}=a\left(x_{3 c+2 k}(t)\right)^{T}, \\
a\left(x_{-c}(t)\right) & =I+t e_{13}, \\
a\left(x_{-c-k}(t)\right) & =I+t e_{12}, \\
a\left(x_{-2 c-k}(t)\right) & =I-t e_{41} .
\end{aligned}
$$

We now fix these representations of the root groups and identify $x_{\phi}(t)$ with the corresponding matrix for the rest of the paper. 
Note that, for each root $\phi,\left\{x_{\phi}(t) \mid t \in A\right\}$ is a one-parameter subgroup of $G_{2}(A)$. So, for instance, for each short root $\phi \in \Phi_{s},\left\{x_{\phi}(t) \mid t \in A\right\} \cup\left\{x_{-\phi}(t) \mid t \in A\right\}$ generates a copy of $E(2, A)$, while $\left\{x_{\phi}(t) \mid \phi \in \Phi_{\ell}, t \in A\right\}$ generates $E(3, A)$.

Commutators. Throughout this paper commutators will be defined by $[x, y]=$ $x^{-1} y^{-1} x y$. Commutator calculations in $G_{2}(A)$ will be facilitated by the following well-known theorem of Chevalley:

(1.1) Theorem (Chevalley). Let $\Phi$ be the root system of $G_{2}$. Suppose that $\phi, \phi^{\prime} \in$ $\Phi$ and that $\phi \neq-\phi^{\prime}$. Then for all $r, s \in A$,

$$
\left[x_{\phi}(r), x_{\phi^{\prime}}(s)\right]=\prod x_{i \phi+j \phi^{\prime}}\left( \pm C_{i j} r^{i} s^{j}\right)
$$

where the product is taken over all $i, j \geq 1$ such that $i \phi+j \phi^{\prime} \in \Phi$ and the $C_{i j}$ are integers from the Cartan matrix. (As usual, the empty product is 1.)

In order to simplify later arguments, we isolate a special subgroup of $G_{2}(A)$. Let $T$ be the diagonal subgroup, the split maximal torus of $G_{2}(A)$. By (A.13), $T$ is the diagonal subgroup of $S L(3, A)$. We set $\mathcal{N}=N_{G_{2}(A)}(T)$; the quotient $\mathcal{N} / T$ is the Weyl group of $G_{2}(A)$, which has order 12 . The group $\mathcal{N}$ includes the monomial matrices in $S L(3, A)$ whose non-zero entries are units in $A$. It is then generated by these and the permutation $(15)(26)(37)(48)$.

The following are representative formulas obtained from (1.1) using the root system of Figure 1. All others can be deduced from the action of the group $\mathcal{N}$.

$$
\begin{aligned}
{\left[x_{k}(s), x_{c}(t)\right] } & =x_{c+k}( \pm s t) x_{2 c+k}\left( \pm s^{2} t\right) x_{3 c+k}\left( \pm s t^{3}\right) x_{3 c+2 k}\left( \pm s^{2} t^{3}\right) ; \\
{\left[x_{3 c+k}(s), x_{k}(t)\right] } & =x_{3 c+2 k}( \pm s t) ; \\
{\left[x_{2 c+k}(s), x_{c}(t)\right] } & =x_{3 c+k}( \pm 3 s t) ; \\
{\left[x_{c+k}(s), x_{c}(t)\right] } & =x_{2 c+k}( \pm 2 s t) x_{3 c+k}\left( \pm 3 s t^{2}\right) x_{3 c+2 k}\left( \pm 3 s^{2} t\right) .
\end{aligned}
$$

Note that the choice of representation of the $x_{\phi}(t)$ 's made above actually determines the signs in these commutator fomulae. Without making the signs explicit, we will later use the fact that they are determined.

We now proceed to derive some basic results about commutators of $E(A)$ normalized subgroups of $G_{2}(A)$. Let $H$ be an $E(A)$-normalized subgroup of $G_{2}(A)$ and let $N=[E(A), H]$. In what follows we shall call two short roots $\phi, \phi^{\prime}$ adjacent if the angle between them is $\pi / 3$. Also, we denote by $X_{\phi}$ the group generated by $\left\{x_{\phi}(t) \mid t \in A\right\}$.

(1.2) Lemma. Suppose that $H$ is an $E(A)$-normalized subgroup with $x_{\phi}(s) x_{\phi^{\prime}}(t)$ $\in H$ for some $s, t \in A$ and some $\phi, \phi^{\prime} \in \Phi_{s}$ with $\phi$ and $\phi^{\prime}$ adjacent. Then $z_{c}(s)$ and $z_{c}(t)$ are in $H$.

Proof. (We actually prove more. We use the fact that $H$ is normalized by $\mathcal{N}$ and by $x_{\sigma}(t)$ for $\sigma \in \Phi_{s}$.)

The group $\mathcal{N}$ is transitive on the 12 ordered pairs of adjacent short roots. So $x_{\sigma}( \pm s) x_{\tau}( \pm t) \in H$ for all $\sigma, \tau \in \Phi_{s}$ which are adjacent. Conjugation by torus elements with \pm 1 on the diagonal allows us to make the signs whatever we wish. In particular, $x_{-c}(s) x_{c+k}(t), x_{2 c+k}(s) x_{c+k}(t)$, and $x_{2 c+k}(s) x_{c}(t) \in H$.

Now we have

$$
x_{-c}(s) x_{c+k}(t)\left[x_{2 c+k}(s) x_{c+k}(t)\right]^{-1} x_{2 c+k}(s) x_{c}(t) \in H .
$$


So $X=x_{-c}(s) x_{c}(t) \in H$. The group $\left\langle X_{-c}, X_{c}\right\rangle=E(2, A)$ and $X$ corresponds to $\left[\begin{array}{cc}1 & s \\ t & 1+s t\end{array}\right]$. Now $H \cap\left\langle X_{-c}, X_{c}\right\rangle$ is normal there, and so the image in $E(2, A)$ contains $\left[\begin{array}{cc}1 & s \\ s & 1+s^{2}\end{array}\right]$ and $\left[\begin{array}{cc}1 & t \\ t & 1+t^{2}\end{array}\right]$ (see $\left.[7]\right)$. The result follows.

(1.3) Lemma. Let $H$ be an $E(A)$-normalized subgroup of $G_{2}(A)$ such that $z_{k}(h) \in$ $H$ for some $h$ in $A$. Then $C(A h) \subseteq H$.

Proof. We have $z_{k}(h) \in H \cap S L(3, A)$. It follows that $E(A ; 0, h A) \subseteq H$. With $t=h t$ and $s=1$ (I) gives

$$
x_{c+k}( \pm h t) x_{2 c+k}( \pm h t) \in H
$$

By Lemma (1.2), $z_{c}( \pm h t) \in H$. By [7], both $z_{c}(h t)$ and $z_{c}(-h t)$ belong to $H$. (Actually the sign is determined, just not explicit. We can get both elements by changing the sign of $t$.)

(1.4) Lemma. Let $H$ be an $E(A)$-normalized subgroup of $G_{2}(A)$ and let $N=$ $[E(A), H]$. Suppose that for some $h \in A, z_{c}(h) \in H$. Then

(i) $E\left(v n_{2}(h A),(h A)_{3}\right) \subseteq N$.

(ii) $C\left(h A,(h A)_{3}\right) \subseteq N$.

(iii) $x_{\phi}(h t) x_{\phi^{\prime}}(h t) \in N$ for any $\phi, \phi^{\prime} \in \Phi_{s}$ and $t \in A$.

Proof. Consider $y=\left[x_{k}(t), z_{c}(h)\right]$. Apply (I) to get

$$
y=x_{c+k}( \pm h t) x_{2 c+k}\left( \pm h^{2} t\right) x_{3 c+k}\left( \pm h^{3} t\right) x_{3 c+2 k}\left( \pm h^{3} t^{2}\right),
$$

an element of $N$. Computing $\left[y, x_{2 c+k}(1)\right]$ shows that $x_{3 c+2 k}(3 h t)$ is in $N$ for every $t \in A$. Since this result can be replicated for all long roots, we have shown that $E(A ; 0,3 h A) \subseteq N$.

Next compute $\left[x_{c+k}(s), z_{c}(h)\right]$ using (IV) to see that $x_{2 c+k}(2 s h) \in N$ for all $s \in A$. It follows that $E(A ; 2 h A, 0) \subseteq N$.

Now observe that $\left[y, x_{k}(1)\right]=x_{3 c+2 k}\left( \pm h^{3} t\right)$, using (II), and hence conclude that $E\left(A ; 0, h^{3} A\right) \subseteq N$. We have now established that $E\left(A ; 0,(h A)_{3}\right) \subseteq N$. In particular, the last two terms in the product giving $y$ are in $N$, so that $y^{\prime}=$ $x_{c+k}( \pm h t) x_{2 c+k}\left( \pm h^{2} t\right)$ is in $N$. From this and Lemma (1.2), it follows that $C(h A, 0)$ $\subseteq N$, and hence that $C\left(h A,(h A)_{3}\right) \subseteq N$. This proves (ii).

The next calculations place more elementary elements in $N$. We know that

$$
x_{c+k}(h t) x_{2 c+k}\left(h^{2} t\right) \in N .
$$

Let $t=t^{2} u$ to get

$$
x_{c+k}\left(h t^{2} u\right) x_{2 c+k}\left(h^{2} t^{2} u\right) \in N .
$$

Since $z_{c}(h t) \in N$, we may replace $h$ by $h t$ and $t$ by $u$ in $\left(^{*}\right)$ to get

$$
x_{c+k}(h t u) x_{2 c+k}\left(h^{2} t^{2} u\right) \in N,
$$

and therefore that $x_{c+k}\left(h\left(t^{2}-t\right) u\right) \in N$. This proves that $E\left(A ; v n_{2}(A) h, 0\right) \subseteq N$.

Now replace $t$ by $h t$ in $\left(^{*}\right)$ and use $\mathcal{N}$ to get $x_{c+k}\left(h^{3} t\right) x_{2 c+k}\left(h^{2} t\right) \in N$. Combining this with $\left(^{*}\right)$, we get $x_{c+k}\left(\left(h^{3}-h\right) t\right) \in N$. But $\left(h^{3}-h\right) t=\left(h^{3}-h^{2}\right) t+\left(h^{2}-h\right) t$, and $h^{3}-h^{2} \in v n_{2}(A) h$, so that $x_{c+k}\left(\left(h^{2}-h\right) t\right) \in N$. It follows that $E\left(A ; v n_{2}(h A), 0\right) \subseteq$ $N$. This proves (i). It is easy to see (iii); this completes the proof.

Lemmas (1.3) and (1.4) immediately yield the following corollary: 
(1.5) Theorem. Let $S, S^{\prime}$ be two subsets of the ring $A$ and consider the subgroup $N=C\left(S, S^{\prime}\right)$ of $G_{2}(A)$. Then

(i) $N=C\left((S)+\left(S^{\prime}\right),\left(S^{\prime}\right)+(S)_{3}\right)$.

(ii) $E\left(A ; v n_{2}\left((S)+\left(S^{\prime}\right)\right),\left(S^{\prime}\right)+(S)_{3}\right) \subseteq N$.

(iii) If $h \in(S)+\left(S^{\prime}\right)$, then $x_{\phi}(h) x_{\phi^{\prime}}(h) \in N$ for any $\phi, \phi^{\prime} \in \Phi_{s}$.

(Here $(S)$ denotes the ideal of A generated by the set $S$.)

Note that the result implies that any subgroup of the form $C\left(S, S^{\prime}\right)$ can be written as $C\left(J, J^{\prime}\right)$, where $J$ and $J^{\prime}$ are ideals of $A$ such that $J_{3} \subseteq J^{\prime} \subseteq J$.

We are now ready for two results concerning $C\left(S, S^{\prime}\right)$ which demonstrate that it plays a fundamental role in describing the normal subgroups of $G_{2}(A)$.

(1.6) Theorem. For subsets $S, S^{\prime}$ of $A$ we have $\left[E(A), C\left(S, S^{\prime}\right)\right]=C\left(S, S^{\prime}\right)$.

Proof. This is clear because (1.3) and (1.4) show that $\left[E(A), C\left(S, S^{\prime}\right)\right]$ contains $C\left(S, S^{\prime}\right)$.

(1.7) Theorem. For subsets $S, S^{\prime}$ of $A$ we have $\left[E(A), E\left(S, S^{\prime}\right)\right]=C\left(S, S^{\prime}\right)$.

Proof. By the previous theorem,

$$
\left[E(A), E\left(A ; S, S^{\prime}\right)\right] \supseteq C\left(S, S^{\prime}\right) .
$$

To obtain the reverse inclusion is to see that the elements of $E(A)$ commute with the elements of $E\left(A ; S, S^{\prime}\right)$ modulo $C\left(S, S^{\prime}\right)$. For this it is sufficient to check whether $x_{\phi}(t)$ commutes with $x_{\sigma}(s)$ and $x_{\tau}\left(s^{\prime}\right)$ modulo $C\left(S, S^{\prime}\right)$ for all $\phi \in \Phi, \sigma \in \Phi_{s}, \tau \in$ $\Phi_{\ell}, t \in A, s \in S$, and $s^{\prime} \in S^{\prime}$. So we want to see that $\left[x_{\phi}(t), x_{\phi^{\prime}}(r)\right] \in C\left(S, S^{\prime}\right)$ with $\phi^{\prime} \in \Phi_{s}$ and $r \in S$ or $\phi^{\prime} \in \Phi_{\ell}$ and $r \in S^{\prime}$.

If $\left[x_{\phi}(t), x_{\phi^{\prime}}(r)\right]=1$, we are done. If not, there exists $w \in \mathcal{N}$ so that

$$
\left[x_{\phi}(t), x_{\phi^{\prime}}(r)\right]^{w}=\left[x_{w(\phi)}(t), x_{w\left(\phi^{\prime}\right)}(r)\right]
$$

is one of the commutators, (I)-(IV).

Simple inspection and application of Theorem (1.5) gives the result.

The $A_{2}$-level of a subgroup. As we have seen in Theorem (1.5), elements of a subgroup $H$ of $G_{2}(A)$ which are products of short-root elementaries make a different contribution to the level ideal than elements which are products of long-root elementaries. Since the long-root elementaries are embedded in the copy of $S L(3, A)$ in $G_{2}(A)$, we may isolate this difference by studying the level ideal of $H \cap S L(3, A)$. We formalize this in the following definition:

(1.8) Definition. Let $\Gamma$ be a subset of the group $G=G_{2}(A)$. We define the $A_{2}$ level of $\Gamma$ to be $\ell z(\Gamma)=\ell(N(\Gamma) \cap S L(3, A))$, where $N(\Gamma)$ is the $E(A)$-normalized subgroup of $G$ generated by $\Gamma$. For a single element $X$ in $G$, we write $\ell z(X)$ for $\ell z(\{X\})$.

The aim of this subsection is to prove that the $A_{2}$-level of $E(A ; J, 0)$ is $J_{3}$. To that end, we begin with some simple lemmas we will need.

(1.9) Lemma. Let $T$ be the torus of $G_{2}(A)$. Let $g \in T, \phi \in \Phi, s \in A$. Then for some unit $\lambda$ of $A$ we have $\left[g, x_{\phi}(s)\right]=x_{\phi}((1-\lambda) s)$.

Proof. We only need observe that $x_{\phi}(A)$ is a one-parameter subgroup and that $x_{\phi}(s)^{g}=x_{\phi}(\lambda s)$, for some unit $\lambda$. (This is easy to see in the matrix representation of $G_{2}(A)$.) 
(1.10) Lemma. Let $J$ be an ideal in a ring $A$ such that $J_{3}=0$. Let $\left[\begin{array}{ll}a & b \\ c & d\end{array}\right]$ be an element of $S L(2, A)$ congruent to the identity modulo $J$, and let $t \in J$. Then

(i)

$$
\begin{aligned}
{\left[\begin{array}{ll}
a & b \\
c & d
\end{array}\right] } & =\left[\begin{array}{cc}
a & 0 \\
0 & a^{-1}
\end{array}\right]\left[\begin{array}{cc}
1 & 0 \\
a c & 1
\end{array}\right]\left[\begin{array}{cc}
1 & a^{-1} b \\
0 & 1
\end{array}\right] \\
& =\left[\begin{array}{cc}
d^{-1} & 0 \\
0 & d
\end{array}\right]\left[\begin{array}{cc}
1 & d b \\
0 & 1
\end{array}\right]\left[\begin{array}{cc}
1 & 0 \\
d^{-1} c & 1
\end{array}\right]
\end{aligned}
$$

(ii)

$$
\begin{aligned}
& {\left[\begin{array}{cc}
1+t & 0 \\
0 & 1-t+t^{2}
\end{array}\right]} \\
& \quad=\left[\begin{array}{cc}
1 & -t^{2} \\
0 & 1
\end{array}\right]\left[\begin{array}{cc}
1 & 0 \\
-t+t^{2} & 1
\end{array}\right]\left[\left[\begin{array}{cc}
1 & 0 \\
-1 & 1
\end{array}\right],\left[\begin{array}{ll}
1 & t \\
0 & 1
\end{array}\right]\right] .
\end{aligned}
$$

(1.11) Lemma. Let $J$ be an ideal in a ring $A$ such that $J_{3}=0$. Then any element of $G(J)$ which is in the center of $S L(3, A)$ is in $E(A ; J, 0)$.

Proof. Let $X \in G(J)$ and suppose that $X$ is in the center of $S L(3, A)$. Then $X=\operatorname{diag}\left(1, \lambda, \lambda, \lambda, 1, \lambda^{-1}, \lambda^{-1}, \lambda^{-1}\right)$ for some $\lambda \in A$ with $\lambda^{3}=1$, by (A.13). Since $X \in G(J)$, we have that $\lambda=1+t$, for some $t \in J$. But $X=\sigma\left(\left[\begin{array}{cc}\lambda & 0 \\ 0 & \lambda^{2}\end{array}\right]\right)$, so part (ii) of (1.10) shows that $X \in E(A ; J, 0)$. This proves the lemma.

The next step toward our goal is to develop a "Gauss"-like decomposition of $E(A ; J, 0)$ under the hypothesis that $J_{3}=0$. For this purpose we set

$$
\begin{aligned}
\Lambda & =\left\{X \in G_{2}(A) \mid X \in G(J) \cap \operatorname{center}(S L(3, A))\right\}, \\
U^{+} & \left.=\left\langle x_{\phi}(s)\right| \phi \in \Phi_{s}, \phi \text { is positive, and } s \in J\right\rangle, \text { and } \\
U^{-} & \left.=\left\langle x_{\phi}(s)\right| \phi \in \Phi_{s}, \phi \text { is negative, and } s \in J\right\rangle,
\end{aligned}
$$

where \langle\rangle indicates the subgroup generated by the listed elements. We first show that $E(A ; J, 0)=\left\langle\Lambda, U^{+}, U^{-}\right\rangle$.

(1.12) Lemma. Let $J$ be an ideal in a ring $A$ such that $J_{3}=0$. With the notation above,

$$
E(A ; J, 0)=\left\langle\Lambda, U^{-}, U^{+}\right\rangle .
$$

Proof. From (1.11) we know that $\Lambda \subseteq E(A ; J, 0)$, and this proves that $\left\langle\Lambda, U^{-}, U^{+}\right\rangle$ $\subseteq E(A ; J, 0)$. To establish the reverse inclusion, it suffices to see that $\left[x_{\rho}(s), x_{\phi}(t)\right] \in$ $\left\langle\Lambda, U^{-}, U^{+}\right\rangle$for all $\rho \in \Phi, \phi \in \Phi_{s}, s \in A$, and $t \in J$. If $\rho \neq-\phi$, this is clear from the commutator formulae (I)-(IV). If $\rho=-\phi$, the result follows from part (i) of (1.10) via one of the embeddings $\sigma_{1}, \sigma_{2}, \sigma_{3}$.

Now for the promised decomposition. (We note that this is a special case of Proposition 2.1 of [3].) 
(1.13) Lemma. Let $J$ be an ideal in a ring $A$ such that $J_{3}=0$. With the notation above,

$$
E(A ; J, 0)=\Lambda U^{-} U^{+} .
$$

Proof. By (1.12), $E(A ; J, 0)=\left\langle\Lambda, U^{+}, U^{-}\right\rangle$so we need only show that $E(A ; J, 0) \subseteq$ $\Lambda U^{-} U^{+}=S$. We claim

(1.14) Lemma. If $\phi \in \Phi_{s}, t \in J, u \in U^{+}$, then $u X_{\phi}(t) \in S$.

Suppose that (1.14) is false. Relative to the lexicographic ordering on the short roots, let $\rho$ be the largest root for which (1.14) is false. Among the elements of $U^{+}$ for which (1.14) is false for $\rho$, choose one that is the product of the smallest number of positive root elementaries for short roots, and let $t \in J$ be chosen with $u x_{\rho}(t)$ not in $S$.

Since $x_{\rho}(t) \in S, u \neq 1$ so that $u=u^{\prime} x_{\sigma}(s)$ for some $u^{\prime} \in U^{+}$(a product of shorter length), $\sigma \in \Phi_{s}, s \in J, \sigma$ positive.

Suppose $\rho=-\sigma$. Then

$$
\begin{aligned}
u x_{\rho}(t) & =u^{\prime} x_{\sigma}(s) x_{-\sigma}(t) \\
& =u^{\prime} R x_{-\sigma}\left(t^{\prime}\right) x_{\sigma}\left(s^{\prime}\right) \\
& =R\left(u^{\prime}\right)^{R} x_{-\sigma}\left(t^{\prime}\right) x_{\sigma}\left(s^{\prime}\right) \\
& =R u^{\prime \prime} x_{-\sigma}\left(t^{\prime}\right) x_{\sigma}\left(s^{\prime}\right),
\end{aligned}
$$

where $R \in \Lambda, s^{\prime}, t^{\prime} \in J$, and where we have used (1.9) and (1.10). Our assumptions give $u^{\prime \prime} x_{-\sigma}\left(t^{\prime}\right) \in S$ and thus $u x_{\rho}(t) \in S$.

If $\rho \neq-\sigma$, then

$$
\begin{aligned}
u x_{\rho}(t) & =u^{\prime} x_{\sigma}(s) x_{\rho}(t) \\
& =u^{\prime} x_{\rho}(t) x_{\sigma}(s)\left[x_{\sigma}(s), x_{\rho}(t)\right] .
\end{aligned}
$$

By our assumptions, $u^{\prime} x_{\rho}(t)=R^{\prime} v^{-} v^{+}$with $R^{\prime} \in \Lambda, v^{-} \in U^{-}$and $v^{+} \in U^{+}$and $v^{+} x_{\sigma}(s)\left[x_{\sigma}(s), x_{\rho}(t)\right] \in S$, completing the proof of (1.14).

It now follows easily from (1.14) that $S U^{-} \subseteq S$. Suppose that $g \in S$ and $R \in \Lambda$. By (1.9) we know that $g^{R} \in S$; but $g R=R \cdot g^{R}$, and we see that $S \cdot \Lambda \subseteq S$. Thus, $S \cdot\left\langle\Lambda, U^{-}, U^{+}\right\rangle \subseteq S$. Now $\left\langle\Lambda, U^{-}, U^{+}\right\rangle=E(A ; J, 0)$ by (1.12), so that $S=E(A ; J, 0)$ and the proof of (1.13) is complete.

(1.15) Theorem. The $A_{2}$-level of $E(A ; J, 0)$ is $J_{3}$.

Proof. Since the $A_{2}$-level of $E(A ; J, 0)$ contains $J_{3}$ by $(1.5)$, it suffices to show that $\ell z(E(A ; J, 0))=0$ when $J_{3}=0$.

Let $s \in \ell z(E(A ; J, 0))$. Then classical results about $S L(3, A)$ show that $x_{k}(s) \in$ $E(A ; J, 0)$. By (1.13), we have $x_{k}(s)=g h$, where $g=R u^{-} x_{c}\left(u_{1}\right)$ and $h=$ $x_{c+k}\left(u_{2}\right) x_{2 c+k}\left(u_{3}\right)$ for some $R \in \Lambda, u^{-} \in U^{-}$, and $u_{1}, u_{2}, u_{3} \in J$. This allows 
us to compute $x_{-3 c-k}(s)$ as follows:

$$
\begin{aligned}
x_{-3 c-k}(s) & =\left[x_{-3 c-2 k}(1), x_{k}(s)\right] \\
& =\left[x_{-3 c-2 k}(1), g h\right] \\
& =\left[x_{-3 c-2 k}(1), h\right]\left[x_{-3 c-2 k}(1), g\right]^{h} \\
& =\left[x_{-3 c-2 k}(1), h\right] .
\end{aligned}
$$

In the matrix representation of $x_{-3 c-k}(s)$, the $(4,3)$-entry is $s$, while the $(4,3)$-entry of $\left[x_{-3 c-2 k}(1), h\right]$ is 0 . This completes the proof.

[Alternatively, one could compute that

$$
\left[x_{-3 c-k}(1),\left[x_{-k}(1),\left[x_{-3 c-2 k}(1), x_{k}(s)\right]\right]\right]=x_{-k}(-s),
$$

while $\left[x_{-3 c-k}(1),\left[x_{-k}(1),\left[x_{-3 c-2 k}(1), h\right]\right]\right]=1$.

A useful homomorphism. In this subsection, we construct an $E(A)$-invariant homomorphism $F$ from a congruence subgroup $G(J)$ to the additive group $J / v n_{2}(J)$ with the property that (1) for $X \in G(J), F(X)$ is a polynomial in the entries of $X$, and (2) for any ideal $J^{\prime}$ such that $J_{3} \subseteq J^{\prime} \subseteq J, \operatorname{ker}\left(\left.F\right|_{E\left(J, J^{\prime}\right)}\right)=C\left(J, J^{\prime}\right)$. The strategy for doing this is based on the observation that for each maximal ideal $\mathcal{M}$ of $A,\left(J / v n_{2}(J)\right)_{\mathcal{M}}$ is either 0 or isomorphic to $\mathbf{Z}_{2}$, the field of two elements. Thus, we first construct the desired homomorphism for the case $A=\mathbf{Z}_{2}, J=A$, and then use local arguments.

The case $A=\mathbf{Z}_{2}, J=A$ : Let $G=G_{2}\left(\mathbf{Z}_{2}\right)$. Let $V$ be an 8-dimensional vector space over $\mathbf{Z}_{2}$ with elements written as row vectors, $v=\left[v_{1}, \ldots, v_{8}\right]$. Set

$$
U=\left\{v \in V \mid v_{1}+v_{5}=1 \text { and }\left[v_{2}, v_{3}, v_{4}\right] \cdot\left[v_{6}, v_{7}, v_{8}\right]=0\right\},
$$

a subset of $V$. Let $u_{1}=[1,0,0,0,0,0,0,0]$. Note that for any $X \in G_{2}\left(\mathbf{Z}_{2}\right), u_{1} X \in U$ by (A.4).

(1.16) Lemma. $U=\left\{u_{1} X \mid X \in G\right\}$ and hence $U X=U$ for any $X \in G$.

Proof. Simple counting gives $|U|=72$. Now $\left|S L\left(3, \mathbf{Z}_{2}\right)\right|=168$ and $|G|=12,096$, so that there are 72 cosets of $S L\left(3, \mathbf{Z}_{2}\right)$ in $G$. If $X_{1}, X_{2} \in G$ with $u_{1} X_{1}=u_{1} X_{2}$, then $X_{1} X_{2}^{-1} \in S L\left(3, \mathbf{Z}_{2}\right)$, by (A.14). The result follows.

(1.17) Lemma. Let $\chi$ be the characteristic function of the set of non-zero vectors in a 3-dimensional vector space over $\mathbf{Z}_{2}$. Define a function $\theta: U \rightarrow \mathbf{Z}_{2}$ by

$$
\theta(v)=v_{5}+\chi\left(v_{2}, v_{3}, v_{4}\right)+\chi\left(v_{6}, v_{7}, v_{8}\right) .
$$

Then the map $\Theta: G \rightarrow \mathbf{Z}_{2}$ given by $\Theta(X)=\theta\left(u_{1} X\right)$ is a homomorphism onto $\mathbf{Z}_{2}$.

Proof. First we show that for any $P$ in $G$ the function $\sigma_{P}: U \rightarrow \mathbf{Z}_{2}$ given by $\sigma_{P}(u)=\theta(u)+\theta(u P)$ is constant. To this end, set $K=\left\{P \in G \mid \sigma_{P}\right.$ is constant $\}$. Note that $P \in K$ if and only if $\sigma_{P}(u)+\sigma_{P}(v)=0$ for every $u, v \in U$.

(1) We show that $K$ is a subgroup of $G$. Let $u, v \in U$ and $P \in K$. We have $0=\sigma_{P}\left(u P^{-1}\right)+\sigma_{P}\left(v P^{-1}\right)=\sigma_{P^{-1}}(u)+\sigma_{P^{-1}}(v)$, so that $P^{-1} \in K$.

If $P_{1}, P_{2} \in K$,

$$
\begin{aligned}
\sigma_{P_{1} P_{2}}(u)+\sigma_{P_{1} P_{2}}(v) & =\sigma_{P_{2}}\left(u P_{1}\right)+\sigma_{P_{1}}(u)+\sigma_{P_{2}}\left(v P_{1}\right)+\sigma_{P_{1}}(v) \\
& =0,
\end{aligned}
$$


and $K$ is a subgroup.

(2) Suppose that $P \in G$ and $a(P)=\left[\begin{array}{ll}1 & 0 \\ 0 & y\end{array}\right]$, where $y$ is a $3 \times 3$ block. Then

$$
\begin{aligned}
\sigma_{P}(u) & =\theta(u)+u_{5}+\chi\left(\left(u_{2}, u_{3}, u_{4}\right) y\right)+\chi\left(\left(u_{6}, u_{7}, u_{8}\right) y^{-T}\right) \\
& =\theta(u)+\theta(u)=0 .
\end{aligned}
$$

This shows that $P \in K$.

(3) Suppose that $\omega=\left[\begin{array}{ll}0 & I \\ I & 0\end{array}\right]$. Then

$$
\sigma_{\omega}(u)=\theta(u)+\left(1-u_{5}\right)+\chi\left(\left(u_{6}, u_{7}, u_{8}\right)\right)+\chi\left(\left(u_{2}, u_{3}, u_{4}\right)\right)=1 .
$$

Hence $\omega \in K$.

(4) If $P=x_{c}(1)$, then

$$
\sigma_{P}(u)=1+\left(u_{2} u_{6}+u_{4} u_{8}\right)\left(1+u_{3}\right)=1+\left(u_{3} u_{7}\right)\left(1+u_{3}\right)=1 .
$$

So $x_{c}(1) \in K$. From (1), (2), and (3), we see that the "Weyl" group $\mathcal{N}$ is contained in $K$ and so $x_{\phi}(1) \in K$ for all roots $\phi$. Thus, $K=G$ and our claim that $\sigma_{P}$ is constant for all $P$ in $G$ is established.

We are now ready to check that $\Theta$ is a homomorphism. Let $P_{1}, P_{2} \in G$. Then

$$
\begin{aligned}
\Theta\left(P_{1} P_{2}\right)=\theta\left(u_{1} P_{1} P_{2}\right) & =\sigma_{P_{2}}\left(u_{1} P_{1}\right)+\theta\left(u_{1} P_{1}\right) \\
& =\sigma_{P_{2}}\left(u_{1}\right)+\theta\left(u_{1} P_{1}\right) \\
& =\Theta\left(P_{2}\right)+\Theta\left(P_{1}\right) .
\end{aligned}
$$

This completes the proof of Lemma (1.17).

We are now ready to tackle the general case.

(1.18) Theorem. Let $A$ be a commutative ring and $J$ an ideal in $A$. Let $f \in$ $Z\left[x_{1}, x_{2}, \ldots, x_{8}\right]$ be any polynomial such that for $u \in U, \theta(u) \equiv f(u) \bmod (2)$. Then the map $F: G(J) \rightarrow J / v n_{2}(J)$ defined by $F(X)=f\left(u_{1} X\right) \bmod \left(v n_{2}(J)\right)$ is an $E(A)$-invariant homomorphism. If $J^{\prime}$ is an ideal with $J_{3} \subseteq J^{\prime} \subseteq J$, then $\operatorname{ker}\left(\left.F\right|_{E\left(J, J^{\prime}\right)}\right)=C\left(J, J^{\prime}\right)$.

Proof. Since the map $F$ is defined by a polynomial in the entries of the first row of $X \in G(J)$, the fact that it is an $E(A)$-invariant homomorphism is just a claim that some polynomial equations hold in $A$. This can be checked locally, i.e., by localizing at a maximal ideal of $A$. Thus, we may assume that $A$ is a local ring with maximal ideal $\mathcal{M}$. If $A / \mathcal{M} \neq \mathbf{Z}_{2}$, then $v n_{2}(A)=A$, so that $v n_{2}(J)=J \cap v n_{2}(A)=J$ and the claim holds trivially. If $A / \mathcal{M} \cong \mathbf{Z}_{2}$, then $v n_{2}(A)=\mathcal{M}$. If $J \subseteq \mathcal{M}$, then $v n_{2}(J)=J$ and the claim is trivial again. Finally, if $J=A$, we have that $J / v n_{2}(J)=A / \mathcal{M} \cong \mathbf{Z}_{2}$, and the claim follows from (1.17).

To verify the second part of the theorem, observe that by $(1.7), C\left(J, J^{\prime}\right)=$ $\left[E(A), E\left(J, J^{\prime}\right)\right]$. Since $F$ is an $E(A)$-invariant map into an abelian group, it is immediate that $C\left(J, J^{\prime}\right) \subseteq \operatorname{ker}\left(\left.F\right|_{E\left(J, J^{\prime}\right)}\right)$.

By (1.5), (1.7), and the fact that all short-root elementary elements $x_{\phi}(t)$ are conjugate under $W$, we have $E\left(J, J^{\prime}\right)=x_{c}(J) C\left(J, J^{\prime}\right)$ and $x_{c}\left(v n_{2}(J)\right) \subseteq C\left(J, J^{\prime}\right)$. Thus, to check that $\operatorname{ker}\left(\left.F\right|_{E\left(J, J^{\prime}\right)}\right) \subseteq C\left(J, J^{\prime}\right)$, we only need to see that if $t \in J$ and $F\left(x_{c}(t)\right)=0$, then $t \in v n_{2}(J)$. But this can be checked locally and modulo $v n_{2}(J)$, so once again the only non-trivial case is when $A=\mathbf{Z}_{2}$ and $J=A$. Now 
$u_{1} x_{c}(t)=[1,0,0,0,0,0,-t, 0]$, as is easily computed from the matrix representation of $x_{c}(t)$. Thus,

$$
0=F\left(x_{c}(t)\right)=\theta(1,0,0,0,0,0,-t, 0)=\chi(0,-t, 0)=t .
$$

The proof of Theorem (1.18) is complete.

We may now derive some consequences of the existence of the homomorphism $F$ of (1.18).

(1.19) Corollary. If $J$ and $J^{\prime}$ are ideals of $A$ such that $J_{3} \subseteq J^{\prime} \subseteq J$, then

$$
E\left(J, J^{\prime}\right) \cap G\left(v n_{2}(J)\right) \subseteq C\left(J, J^{\prime}\right) .
$$

Proof. Let $X \in E\left(J, J^{\prime}\right) \cap G\left(v n_{2}(J)\right)$. Then $X \equiv I \bmod \left(v n_{2}(J)\right)$ implies that $F(X)=0$, and Theorem (1.18) shows that $X \in C\left(J, J^{\prime}\right)$, which completes the proof.

(1.20) Lemma. If $J$ and $J^{\prime}$ are ideals of $A$ such that $J_{3} \subseteq J^{\prime} \subseteq J$, then

$$
\left[E(A), G\left(J, J^{\prime}\right)\right] \subseteq E\left(J, J^{\prime}\right) .
$$

Proof. By a result of Vaserstein [16], $\left[E(A), G\left(J^{\prime}\right)\right] \subseteq E\left(J^{\prime}\right)$. By definition, $G\left(J, J^{\prime}\right)$ $=E\left(J, J^{\prime}\right) G\left(J^{\prime}\right)$, and since $J_{3} \subseteq J^{\prime}$ we have

$$
G\left(J, J^{\prime}\right)=E\left(J, J_{3}\right) G\left(J^{\prime}\right)=E(J, 0) G\left(J^{\prime}\right) .
$$

Putting these together, we have

$$
\begin{aligned}
{\left[E(A), G\left(J, J^{\prime}\right)\right] } & =\left[E(A), G\left(J^{\prime}\right)\right]\left[E(A), E\left(J, J_{3}\right)\right] \\
& \subseteq E\left(J^{\prime}\right) E\left(J, J_{3}\right) \\
& =E\left(J, J^{\prime}\right) .
\end{aligned}
$$

(1.21) Theorem. Let $J$ and $J^{\prime}$ be ideals of $A$ such that $J_{3} \subseteq J^{\prime} \subseteq J$. If $R \in$ $G(A), X \in G\left(J, J^{\prime}\right)$, and $Y=[R, X] \in E\left(J, J^{\prime}\right)$, then $Y \in C\left(J, J^{\prime}\right)$. Consequently,

$$
\left[E(A), G\left(J, J^{\prime}\right)\right]=C\left(J, J^{\prime}\right) .
$$

Proof. To show that $Y \in C\left(J, J^{\prime}\right)$, it suffices to check that $F(Y)=0$, where $F$ is the homomorphism of (1.18). Since $F$ is defined by a polynomial, this may be checked locally. As before, we may also assume that $v n_{2}(J)=0$, so that the only possible non-trivial case is $A=\mathbf{Z}_{2}, J=A$. Then $J^{\prime}=A$ as well, and $G\left(J, J^{\prime}\right)=G(A)=E(A)$. Thus, $Y \in[E(A), E(A)]=C(A)$ by (1.7)], and (1.18) shows that $F(Y)=0$, as required.

The second assertion of the theorem follows from the first upon using (1.20). This completes the proof of (1.21).

\section{A new Characterization of $G_{2}(A)$}

In this section we begin with a matrix characterization of $G_{2}(A)$. We adopt the point of view of the appendix, in which $G_{2}(A)=\operatorname{Aut}_{A}(\mathcal{O}(A))$, where $\mathcal{O}(A)$ is the split Cayley algebra (split octonions) over $A$. Since $\mathcal{O}(A)$ is a rank 8 free module over $A$, we fix a basis $u_{1}=e_{11}, \ldots, u_{8}=g_{21}$, as in the appendix, and represent elements $X$ of $G_{2}(A)$ as invertible $8 \times 8$ matrices relative to this fixed basis. We shall freely use the results in the appendix. 
Recall that for $X \in G L(8, A)$ we write $X=\left[a_{i j}\right]=\left[\begin{array}{ll}a & b \\ c & d\end{array}\right]$ where $a, b, c, d$ are $4 \times 4$ blocks which we also denote by $a(X), b(X), c(X), d(X)$. As noted in the appendix, $X$ leaves the bilinear form $N(x, y)$ on $\mathcal{O}(A)$ invariant if and only if $X^{-1}=\left[\begin{array}{ll}d^{T} & b^{T} \\ c^{T} & a^{T}\end{array}\right]$. Also recall that for $\alpha \in \mathcal{O}(A), L_{\alpha}$ is the $8 \times 8$ matrix representing left multiplication by $\alpha$ relative to our fixed basis.

(2.1) Theorem. Let $A$ be a commutative ring, and let $X=\left[\begin{array}{ll}a & b \\ c & d\end{array}\right] \in G L(8, A)$. Then $X \in G_{2}(A)$ if and only if the following conditions hold:

(i) $X^{-1}=\left[\begin{array}{ll}d^{T} & b^{T} \\ c^{T} & a^{T}\end{array}\right]$

(ii) $X$ fixes the identity element 1 of $\mathcal{O}(A)$,

(iii) $X^{-1} L_{e_{11}} X=L_{e_{11} X}$, and

(iv) $\operatorname{det}(a)=a_{11}^{2}$ and $\operatorname{det}(b)=a_{15}^{2}$.

Proof of the necessity of (i)-(iv). From the appendix, (A.2) proves the necessity of (iii), and (A.3) proves the necessity of (i), while the necessity of (ii) is clear. So it only remains to show that if $X \in G_{2}(A)$, then (iv) holds. This can be checked locally, so we may proceed under the assumption that $A$ is a local ring.

From (A.4) we know that $a_{11}+a_{15}=1$, and since $A$ is local, either $a_{11}$ or $a_{15}$ is a unit. But $P=\left[\begin{array}{ll}0 & I \\ I & 0\end{array}\right]$ is in $G_{2}(A)$ and $X P=\left[\begin{array}{ll}b & a \\ d & c\end{array}\right]$, so we may now proceed under the assumption that $a_{11}=\rho$ is a unit.

Now $a d^{T}=\rho I$, so $a$ is invertible, and hence $b=\left(b a^{T}\right) a^{-T}$. We now note that it suffices to prove that $\operatorname{det}(a)=\rho^{2}$, for then $\operatorname{det}(b)=\operatorname{det}\left(b a^{T}\right) \cdot \rho^{-2}$. But, from (A.6) we have

$$
\operatorname{det}\left(b a^{T}\right)=\operatorname{det}\left(a b^{T}\right)=\left(a_{21} a_{61}+a_{31} a_{71}+a_{41} a_{81}\right)^{2} .
$$

On the other hand, computing the first entry of $a^{T} c$, we get $0=\rho(1-\rho)+a_{21} a_{61}+$ $a_{31} a_{71}+a_{41} a_{81}$. Thus, $\operatorname{det}\left(b a^{T}\right)=\rho^{2}(1-\rho)^{2}$, and hence $\operatorname{det}(b)=(1-\rho)^{2}$, as required.

It remains to verify that $\operatorname{det}(a)=\rho^{2}$. Set $\lambda=\operatorname{det}(a)$. By (A.7),

$$
\lambda=\operatorname{det}\left[\begin{array}{lll}
a_{22} & a_{23} & a_{24} \\
a_{32} & a_{33} & a_{34} \\
a_{42} & a_{43} & a_{44}
\end{array}\right] .
$$

Denote this $3 \times 3$ matrix by $M$. Then

$$
M^{-1}\left[\begin{array}{lll}
1 & 0 & 0 \\
0 & 1 & 0 \\
0 & 0 & \lambda
\end{array}\right]=L
$$

is in $S L(3, A)$ and so there is a unique matrix $Y$ in $G_{2}(A)$ such that

$$
a(Y)=\left[\begin{array}{cccc}
1 & 0 & 0 & 0 \\
0 & & & \\
0 & & L & \\
0 & & &
\end{array}\right] .
$$


We may now replace $X$ by $X Y$, for this doesn't change $\operatorname{det}(a)$. Therefore, we may now proceed under the assumption that

$$
a(X)=\left[\begin{array}{cccc}
\rho & a_{12} & a_{13} & a_{14} \\
a_{21} & 1 & 0 & 0 \\
a_{31} & 0 & 1 & 0 \\
a_{41} & 0 & 0 & \lambda
\end{array}\right] .
$$

From (A.9) we have that $a_{88}=-m_{2318}+m_{2323}$. Given the form of $a(X)$ above, this reduces to

$$
a_{88}=a_{31} a_{28}-a_{21} a_{38}+1
$$

We next compute

$$
a^{-T}=\lambda^{-1} \operatorname{adj}(a)^{T}=\left[\begin{array}{cccc}
1 & * & * & -\lambda^{-1} a_{41} \\
-a_{12} & * & * & \lambda^{-1} a_{12} a_{41} \\
-a_{13} & * & * & \lambda^{-1} a_{13} a_{41} \\
-\lambda^{-1} a_{14} & * & * & \lambda^{-1}\left(\rho-a_{12} a_{21}-a_{13} a_{31}\right)
\end{array}\right] .
$$

Since $b=\left(b a^{T}\right) a^{-T}=-\left(a b^{T}\right) a^{-T}$, we may compute $a_{28}=b_{24}$ and $a_{38}=b_{34}$ using the matrix for $a b^{T}$ in (A.6) and the last column of $a^{-T}$. Carrying this out and using the relation

$$
0=\rho(1-\rho)+a_{21} a_{61}+a_{31} a_{71}+a_{41} a_{81}
$$

we get

$$
a_{31} a_{28}-a_{21} a_{38}=-\lambda^{-1}\left[\rho(1-\rho)\left(a_{12} a_{21}+a_{13} a_{31}\right)+\rho\left(a_{21} a_{61}+a_{31} a_{71}\right)\right] .
$$

Next, since $d=\rho a^{-T}, a_{88}=\rho \lambda^{-1}\left(\rho-a_{12} a_{21}-a_{13} a_{31}\right), a_{65}=-\rho a_{12}$, and $a_{75}=-\rho a_{13}$. By (A.4) we get that $a_{61}=\rho a_{12}$ and $a_{71}=\rho a_{13}$. Substituting into the equation for $a_{88}$ above and multiplying by $\lambda$, we get

$$
\lambda=\rho^{2},
$$

which was to be proved. This completes the proof of the necessity in (2.1).

We next show how to prove the sufficiency of (i)-(iv) given that necessity holds. To do this, we must first establish some consequences of (i)-(iv) and use them to prove a factorization theorem.

(2.2) Lemma. Let $X \in G L(8, A)$ and assume that (i)-(iv) hold for $X$. Then (A.4) holds for $X, d^{T} a=a_{11} I$, the entries of $d^{T} b$ and $c^{T} a$ are as in (A.6), and (A.7) and (A.8) hold for $X$. Consequently, if $X, X^{\prime}$ both satisfy (i)-(iv), $a(X)=a\left(X^{\prime}\right)$, and $a_{11}$ is a unit, then $X=X^{\prime}$.

Proof. That (A.4) holds follows from (ii), which implies that $X^{-1}$ fixes 1 also. Note that the matrix of $L_{e_{11}}$ relative to the basis $u_{1}, \ldots, u_{8}$ we are using for $\mathcal{O}(A)$ is 
$\left[\begin{array}{ll}I & 0 \\ 0 & 0\end{array}\right]$. Now

$$
X^{-1} L_{e_{11}} X=\left[\begin{array}{cc}
d^{T} & b^{T} \\
c^{T} & a^{T}
\end{array}\right]\left[\begin{array}{ll}
I & 0 \\
0 & 0
\end{array}\right]\left[\begin{array}{cc}
a & b \\
c & d
\end{array}\right]=\left[\begin{array}{cc}
d^{T} a & d^{T} b \\
c^{T} a & c^{T} b
\end{array}\right] .
$$

On the other hand, $L_{e_{11} X}=\sum_{j=1}^{8} a_{1_{j}} L_{u_{j}}$. Writing out the matrices for the $L_{u_{j}}$ 's, summing and using (iii) shows that $d^{T} a, d^{T} b, c^{T} a$ are as claimed. If $a_{11}$ is a unit, then (A.7) and (A.8) are proved exactly as in the appendix.

Next we establish that certain matrices always belong to the elementary group $E(A)$ in $G_{2}(A)$.

(2.3) Lemma. Let $r, s, t \in A$. Then $E(A)$ contains the matrix

$$
X(r, s, t)=\left[\begin{array}{cccccccc}
1 & 0 & 0 & 0 & 0 & -r & -s & -t \\
-r & 1 & 0 & 0 & r & r^{2} & r s & r t \\
-s & 0 & 1 & 0 & s & r s & s^{2} & s t \\
-t & 0 & 0 & 1 & t & r t & s t & t^{2} \\
0 & 0 & 0 & 0 & 1 & r & s & t \\
0 & 0 & t & -s & 0 & 1 & 0 & 0 \\
0 & -t & 0 & r & 0 & 0 & 1 & 0 \\
0 & s & -r & 0 & 0 & 0 & 0 & 1
\end{array}\right] .
$$

Proof. Set

$$
\begin{aligned}
R & =x_{3 c+2 k}\left(2 r^{2} s\right) x_{3 c+k}\left(r s^{2}\right), \\
S_{1} & =x_{c}(s) x_{c+k}(r) x_{2 c+k}(r s), \\
S_{2} & =x_{-2 c-k}\left(t+r s t^{2}\right) x_{-c-k}(-s t) x_{-c}(r t),
\end{aligned}
$$

and let $T$ be the unique element of $S L(3, A)$ such that

$$
a(T)=\left[\begin{array}{cccc}
1 & 0 & 0 & 0 \\
0 & 1-r s t & r^{2} t & 0 \\
0 & -s^{2} t & 1+r s t & 0 \\
0 & r^{2} s^{3} t^{4}-2 s t^{2}-2 r s^{2} t^{3} & -r^{3} s^{2} t^{4}+2 r t^{2}+r^{2} s t^{3} & 1
\end{array}\right] .
$$

Then direct multiplication gives $R S_{1} S_{2} T=X(r, s, t)$.

Note that $R, S_{1}, S_{2}$ are clearly in $E(A)$ and that $T$ is in $S L(2, A) E(3, A)$. But the Mennicke symbol of $T$ is trivial, so $T \in E(3, A)$. [In fact, it is easy to see directly that $T \in E(3, A)$.] This proves that $X(r, s, t) \in E(A)$.

In order to state the next few results we adopt the following notation: If $X_{1}, X_{2}$ are the unique matrices in $G L(8, A)$ which satisfy (i)-(iv) and have upper left blocks $a\left(X_{1}\right), a\left(X_{2}\right)$, respectively, then we write $a\left(X_{1}\right) \circ a\left(X_{2}\right)$ for $a\left(X_{1} X_{2}\right)$. 


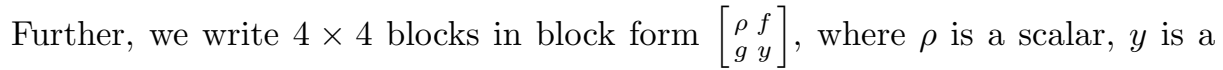
$3 \times 3$ block, and $f, g$ are appropriate vectors.

(2.4) Lemma. For a row $v$ and a column $u$ with entries in $A$,

$$
\left(\begin{array}{ll}
1 & v \\
0 & 1
\end{array}\right) \circ\left(\begin{array}{ll}
1 & 0 \\
u & 1
\end{array}\right)=\left(\begin{array}{cc}
\sigma & v \\
u & H
\end{array}\right)
$$

where $\sigma=1+v u$ and $H=\sigma I-u v$.

Proof. By (2.2), (2.3), and the "transpose" of (2.3), there are unique matrices in $G_{2}(A)$ with upper left blocks $\left(\begin{array}{ll}1 & v \\ 0 & 1\end{array}\right)$ and $\left(\begin{array}{ll}1 & 0 \\ u & 1\end{array}\right)$. By the necessity in $(2.1)$, they satisfy (i)-(iv). Now multiply to get the result.

We note that $\operatorname{det} H=\sigma^{2}$.

(2.5) Lemma. Let $X$ be a matrix in $G L(8, A)$ for which (i)-(iv) hold and for which $a(X)=\left[\begin{array}{ll}\rho & f \\ g & y\end{array}\right]$. If $\rho$ is a unit, then

$$
\left[\begin{array}{ll}
\rho & f \\
g & y
\end{array}\right]=\left[\begin{array}{cc}
1 & f y^{-1} \\
0 & 1
\end{array}\right] \circ\left[\begin{array}{ll}
1 & 0 \\
g & 1
\end{array}\right] \circ\left[\begin{array}{cc}
1 & 0 \\
0 & \left(\rho I-g f y^{-1}\right)^{-1} y
\end{array}\right] .
$$

Proof. Suppose that $\sigma$ is a unit in (2.4), and let $L$ be an element of $G L(3, A)$ with $\operatorname{det}(L)=\sigma^{2}$. Then $M=(\sigma \cdot I-u v)^{-1} L$ is in $S L(3, A)$. From (2.4), we get

$$
\left(\begin{array}{ll}
1 & v \\
0 & 1
\end{array}\right) \circ\left(\begin{array}{ll}
1 & 0 \\
u & 1
\end{array}\right) \circ\left(\begin{array}{cc}
1 & 0 \\
0 & M
\end{array}\right)=\left(\begin{array}{cc}
\sigma & v L \\
u & L
\end{array}\right),
$$

because $v(\sigma I-u v)=\sigma v-v u v=v$.

Now take $u=g, v=f y^{-1}$, and $L=y$. Then $\sigma=1+v u=1+f y^{-1} g$. We will show that $\rho=1+f y^{-1} g$ so that $\sigma=\rho$ is a unit, and this will finish the proof.

By (iv), $\rho^{2}=\operatorname{det} a(X)$ and by $(2.2), \rho^{2}=\operatorname{det} y$. Thus,

$$
\begin{aligned}
\rho^{2} & =\operatorname{det}(a(X))=\rho \operatorname{det}\left(y-\rho^{-1} g f\right)=\rho\left(\operatorname{det}(y)-\rho^{-1} \operatorname{tr}(\operatorname{adj}(y) g f)\right) \\
& =\rho(\operatorname{det}(y))-\operatorname{tr}(\operatorname{adj}(y) g f) .
\end{aligned}
$$

Therefore,

$$
f \operatorname{adj}(y) g=\operatorname{tr}(f \operatorname{adj}(y) g)=\operatorname{tr}(\operatorname{adj}(y) g f)=\rho^{3}-\rho^{2},
$$

giving $f y^{-1} g=\rho-1$ and $\rho=1+f y^{-1} g$.

We are now ready to prove the sufficiency in (2.1).

(2.6) Corollary. Sufficiency holds in (2.1).

Proof. Let $X \in G L(8, A)$ satisfy (i)-(iv). We must verify that the polynomial relations in $\mathcal{P}$ hold. This can be checked locally, so we may assume that $A$ is a local ring. By (A.4), $a_{11}+a_{15}=1$, so either $a_{11}$ or $a_{15}$ is a unit. If $a_{11}$ is not a unit, then we replace $X$ by $X^{\prime}=X\left[\begin{array}{ll}0 & I \\ I & 0\end{array}\right]$. Since $\left[\begin{array}{ll}0 & I \\ I & 0\end{array}\right] \in G_{2}(A)$, it suffices to show that $X^{\prime} \in G_{2}(A)$. On the other hand, it is easy to check that (i)-(iv) still hold for $X^{\prime}$. So, we may now proceed under the assumption that $a_{11}$ is a unit.

By (2.5),

$$
a(X)=\left[\begin{array}{cc}
1 & f y^{-1} \\
0 & 1
\end{array}\right] \circ\left[\begin{array}{ll}
1 & 0 \\
g & 1
\end{array}\right] \circ\left[\begin{array}{cc}
1 & 0 \\
0 & M
\end{array}\right] \text {, with } M \in S L(3, A) .
$$


By $(2.3), X \in E(A) S L(3, A) \subseteq G_{2}(A)$.

This completes the proof of Theorem (2.1).

We close this section with a factorization to be used in the sequel, under the hypothesis that $J_{3}=0$. The factorization appears in the proof of the next lemma.

(2.7) Lemma. Let $J$ be an ideal in a ring $A$ such that $J_{3}=0$. Then $G(J)=$ $E(J)$.

Proof. Let $t_{1}, t_{2}, t_{3} \in J$. Set

$$
\begin{aligned}
& r_{1}=-t_{2}+t_{1} t_{2}^{2} t_{3}, \\
& r_{2}=-t_{1}-t_{1}^{2} t_{2} t_{3}, \\
& r_{3}=t_{1} t_{2}, \\
& r_{4}=t_{1} t_{3}+t_{1}^{2} t_{2} t_{3}^{2}, \\
& r_{5}=-t_{2} t_{3}+t_{1} t_{2}^{2} t_{3}^{2}, \text { and } \\
& r_{6}=-t_{3}-t_{1} t_{2} t_{3}^{2} .
\end{aligned}
$$

We define a matrix $S\left(t_{1}, t_{2}, t_{3}\right)$ in $E(J)$ by

$$
S\left(t_{1}, t_{2}, t_{3}\right)=x_{c}\left(r_{1}\right) x_{c+k}\left(r_{2}\right) x_{2 c+k}\left(r_{3}\right) x_{-c}\left(r_{4}\right) x_{-c-k}\left(r_{5}\right) x_{-2 c-k}\left(r_{6}\right) .
$$

Then

$$
a\left(S\left(t_{1}, t_{2}, t_{3}\right)\right)=\left[\begin{array}{cccc}
1 & 0 & 0 & 0 \\
t_{1} & & & \\
t_{2} & & B & \\
t_{3} & & &
\end{array}\right]
$$

where

$$
B=\left[\begin{array}{ccc}
1-t_{1} t_{2} t_{3}-t_{1}^{2} t_{2}^{2} t_{3}^{2} & t_{1}^{2} t_{3} & -t_{1}^{2} t_{2} \\
-t_{2}^{2} t_{3} & 1+t_{1} t_{2} t_{3}-t_{1}^{2} t_{2}^{2} t_{3}^{2} & t_{1} t_{2}^{2} \\
t_{2} t_{3}^{2} & -t_{1} t_{3}^{2} & 1
\end{array}\right] .
$$

Next, we set $L\left(t_{1}, t_{2}, t_{3}\right)$ equal to the unique matrix in $G_{2}(A)$ such that

$$
a\left(L\left(t_{1}, t_{2}, t_{3}\right)\right)=\left[\begin{array}{cccc}
1 & 0 & 0 & 0 \\
0 & & & \\
0 & & B^{-1} & \\
0 & & &
\end{array}\right] .
$$

Then $L\left(t_{1}, t_{2}, t_{3}\right) \in S L(3, A ; J)$, and

$$
\left[\begin{array}{cccc}
1 & 0 & 0 & 0 \\
t_{1} & 1 & 0 & 0 \\
t_{2} & 0 & 1 & 0 \\
t_{3} & 0 & 0 & 1
\end{array}\right]=a\left(S\left(t_{1}, t_{2}, t_{3}\right)\right) \circ a\left(L\left(t_{1}, t_{2}, t_{3}\right)\right) .
$$


This proves that $X\left(-t_{1},-t_{2},-t_{3}\right)=S\left(t_{1}, t_{2}, t_{3}\right) L\left(t_{1}, t_{2}, t_{3}\right)$, a factorization in which the first factor is a product of short root elementaries and the second is in $S L(3, A ; J)$.

It is clear that $X\left(-t_{1},-t_{2},-t_{3}\right)^{T}=L\left(t_{1}, t_{2}, t_{3}\right)^{T} S\left(t_{1}, t_{2}, t_{3}\right)^{T}$ provides a similar factorization for the matrix with upper left block $\left[\begin{array}{ll}1 & t \\ 0 & I\end{array}\right], t=\left[t_{1}, t_{2}, t_{3}\right]$. Since $x_{\phi}(r)=$ $x_{-\phi}(-r)^{T}$ for any short root $\phi, S\left(t_{1}, t_{2}, t_{3}\right)^{T}$ is still a product involving only short roots.

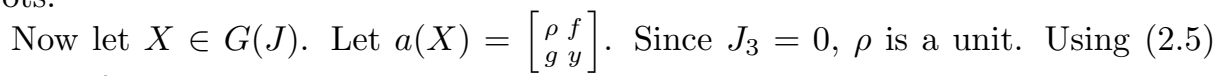
and the factorizations in the previous paragraphs, we have

$$
X=L\left(f y^{-1}\right)^{T} S\left(f y^{-1}\right)^{T} S(g) L(g) Y
$$

where

$$
a(Y)=\left[\begin{array}{cc}
1 & 0 \\
0 & \left(\rho I-g f y^{-1}\right)^{-1} y
\end{array}\right] .
$$

This completes the proof and provides a useful formula.

The proof of (2.7) actually yields a slightly sharper result.

(2.9) Corollary. Let $J$ be an ideal in a commutative ring $A$. Then $G(J)=$ $E(3, A ; J) G\left(J, J_{3}\right)$. [Note that here $E(3, A ; J)$ is merely the subgroup of $S L(3, A)$ and is not the $E(A)$-normalized subgroup $E(0, J)$ of $G_{2}(A)$ which it generates.]

Proof. Since $G\left(J, J_{3}\right)=E(J, 0) G\left(J_{3}\right)$ by definition, it suffices to prove the result modulo $J_{3}$. Thus, we may assume that $J_{3}=0$. Then $J$ satisfies the relative stable range condition $S R_{2}(A, J)$, so that $S L(3, A ; J)=E(3, A ; J)$ [4]. This means that in (2.8) the matrices $L\left(f y^{-1}\right)^{T}, L(g)$, and $Y$ all belong to $E(3, A ; J)$, while $S\left(f y^{-1}\right)^{T}$ and $S(g)$ are in $E(J, 0)$. Then rewriting $(2.8)$ in the form

$$
X=\left(L S\left(f y^{-1}\right)^{T} S(g) L^{-1}\right) L\left(f y^{-1}\right)^{T} L(g) Y,
$$

where $L=L\left(f y^{-1}\right)^{T}$, shows that $X \in E(J, 0) E(3, A ; J)$. This completes the proof of (2.9).

\section{THE MAIN THEOREM}

In this section we prove the main theorem, Theorem (3.6), characterizing the $E(A)$-normalized subgroups of $G_{2}(A)$. It says that a subgroup $H$ is $E(A)$-normalized if and only if there are ideals $J, J^{\prime}$ of $A$ satisfying $J_{3} \subseteq J^{\prime} \subseteq J$ such that $C\left(J, J^{\prime}\right) \subseteq H \subseteq G\left(J, J^{\prime}\right)$. We also show that $C\left(J, J^{\prime}\right)$ and $G\left(J, J^{\prime}\right)$ are normal subgroups of $G_{2}(A)$ and that for any such sandwiched subgroup $H,[E(A), H]=$ $C\left(J, J^{\prime}\right)$.

This will be accomplished by the method of reduction. The first lemma sets this up.

(3.1) Lemma (Reduction Lemma). Let $A$ be a commutative ring, and let $H$ be an $E(A)$-normalized subgroup of $G_{2}(A)$. Let $J_{0}$ be an ideal of $A$ such that $C\left(J_{0}\right) \subseteq H$. If $J_{1}, J_{2}$ are ideals such that $C\left(J_{1}, J_{2}\right) \subseteq H G\left(J_{0}\right)$, then $C\left(J_{1}, J_{2}\right) \subseteq H$. (This says that to show $C\left(J_{1}, J_{2}\right) \subseteq H$, it suffices to do so modulo $J_{0}$.) 
Proof. We have that

$$
\begin{aligned}
C\left(J_{1}, J_{2}\right) & =\left[E(A), C\left(J_{1}, J_{2}\right)\right] \subseteq\left[E(A), H G\left(J_{0}\right)\right] \\
& =[E(A), H]\left[E(A), G\left(J_{0}\right)\right] \subseteq H C\left(J_{0}\right) \subseteq H,
\end{aligned}
$$

where we have used (1.6) and (1.21).

Now for a crucial observation about the $A_{2}$-level of an $E(A)$-normalized subgroup $H$ : if $J^{\prime}=\ell z(H)$, then $C\left(J^{\prime}\right) \subseteq H$, so that we can use reduction (3.1) with $J_{0}=J^{\prime}$. To see this, note that $J^{\prime}=\ell(H \cap S L(3, A))$. Classical results about $E(3, A)$ normalized subgroups of $S L(3, A)$ tell us that $E\left(0, J^{\prime}\right) \subseteq H$. Then $C\left(0, J^{\prime}\right) \subseteq H$; but $C\left(0, J^{\prime}\right)=C\left(J^{\prime}, J^{\prime}\right)=C\left(J^{\prime}\right)$ by $(1.5)$.

The remaining lemmas will set us up for a proof of the main theorem by reduction modulo $J^{\prime}=\ell z(H)$.

(3.2) Lemma. Let $A$ be a commutative ring, and let $H$ be an $E(A)$-normalized subgroup of $G_{2}(A)$ such that $\ell z(H)=0$. If $h$ is an element of $A$ such that $x_{c}(h) \in$ $H$, then $h^{3}=3 h=0$ and $E(h A, 0) \subseteq[E(A), H]$.

Proof. By (1.4), we have that $E\left(v n_{2}(h A),(h A)_{3}\right) \subseteq[E(A), H] \subseteq H$. Since $\ell z(H)=$ 0 , we immediately have $(h A)_{3}=0$, proving that $h^{3}=3 h=0$. But then $h A=$ $2 h A \subseteq v n_{2}(h A)$, so that $E(h A, 0) \subseteq[E(A), h]$ follows.

(3.3) Lemma. Let $A$ be a commutative ring, and let $H$ be an $E(A)$-normalized subgroup of $G_{2}(A)$ such that $\ell z(H)=0$. Suppose that $H$ contains an element $X$ of the form

$$
X=x_{c}\left(u_{1}\right) x_{c+k}\left(u_{2}\right) x_{2 c+k}\left(u_{3}\right) x_{k}\left(u_{4}\right) x_{3 c+k}\left(u_{5}\right) x_{3 c+2 k}\left(u_{6}\right) .
$$

Then $u_{4}=u_{5}=u_{6}=0$ and $x_{c}\left(u_{1}\right), x_{c}\left(u_{2}\right), x_{c}\left(u_{3}\right) \in[E(A), H]$.

Proof. First observe that $\left[x_{3 c+k}(1), X\right]=x_{3 c+2 k}\left(-u_{4}\right)$, so that $u_{4} \in \ell z(H)$ and hence $u_{4}=0$. Next, let

$$
y=\left[x_{-k}(1), X\right]=x_{c+k}\left(u_{2}\right) x_{2 c+k}\left(-u_{2}^{2}\right) x_{3 c+k}\left(u_{6}-3 u_{2} u_{3}-u_{2}^{3}\right) x_{3 c+2 k}\left(-u_{2}^{3}\right) .
$$

Then $\left[x_{2 c+k}(1), y\right]=x_{3 c+k}\left(3 u_{2}\right)$, so that $3 u_{2}=0$. Likewise, we have $\left[x_{c+k}(1), y\right]=$ $x_{2 c+k}\left(2 u_{2}\right)$, so that $x_{2 c+k}\left(u_{2}\right) \in[E(A), H]$.

Applying the action of $\mathcal{N}$ shows that $x_{c}\left(u_{2}\right), x_{c+k}\left(u_{2}\right) \in[E(A), H]$, as well. Lemma (3.2) shows that $u_{2}^{3}=0$ and that $x_{2 c+k}\left(-u_{2}^{2}\right) \in[E(A), H]$, and since $y \in[E(A), H]$, we conclude that $x_{3 c+k}\left(u_{6}\right)$ is in $[E(A), H]$. It follows that $u_{6}=0$.

Next, let $z=x_{c}\left(u_{1}\right) x_{2 c+k}\left(u_{3}\right) x_{3 c+k}\left(u_{5}\right)$. Since $u_{4}=u_{6}=0$ and $X, x_{c+k}\left(u_{2}\right) \in$ $H$, we have that $z \in H$. Form the commutator

$$
R=\left[x_{k}(1), z\right]=x_{c+k}\left(u_{1}\right) x_{2 c+k}\left(u_{1}^{2}\right) x_{3 c+k}\left(u_{1}^{3}\right) x_{3 c+2 k}\left(u_{1}^{3}+u_{5}-3 u_{1} u_{3}\right) .
$$

Applying the first part of the proof to $R$ we see that $x_{c+k}\left(u_{1}\right) \in[E(A), H]$, and by (3.2) that $u_{1}^{3}=3 u_{1}=0$ and $x_{2 c+k}\left(u_{1}^{2}\right) \in[E(A), H]$. It follows that $x_{3 c+2 k}\left(u_{5}\right) \in H$, so that $u_{5}=0$.

We now have $X=x_{c}\left(u_{1}\right) x_{c+k}\left(u_{2}\right) x_{2 c+k}\left(u_{3}\right)$, and hence that $x_{2 c+k}\left(u_{3}\right) \in H$. Applying (3.2) once more completes the proof.

(3.4) Lemma. Let $A$ be a commutative ring and let $H$ be an $E(A)$-normalized subgroup of $G_{2}(A)$ such that $\ell z(H)=0$. Suppose that $X \in H$ and that the upper 
left block of $X$ has the form

$$
a(X)=\left[\begin{array}{cccc}
1 & 0 & a_{13} & a_{14} \\
a_{21} & 1 & a_{23} & a_{24} \\
0 & 0 & a_{33} & a_{34} \\
0 & 0 & a_{43} & a_{44}
\end{array}\right] .
$$

Then

$$
a_{34}=a_{43}=a_{23}+a_{13}^{2} a_{14}+a_{21} a_{13}=a_{24}-a_{13} a_{14}^{2}-2 a_{21} a_{14}=0,
$$

and $a_{33}=a_{44}=1$. Furthermore, $x_{c}\left(a_{13}\right), x_{c}\left(a_{14}\right), x_{c}\left(a_{21}\right), x_{c}\left(a_{23}\right)$, and $x_{c}\left(a_{24}\right)$ are all in $[E(A), H]$.

Proof. A computation shows that $\left[x_{3 c+2 k}(1), X\right]=x_{k}\left(a_{43}\right) x_{3 c+2 k}\left(a_{44}-1\right)$. It now follows from (3.3) that $a_{43}=0$ and $a_{44}=1$. Since $\operatorname{det}(a(X))=1$, we also get that $a_{33}=1$. With this information in hand we compute $\left[x_{k}(1), X\right]=x_{3 c+2 k}\left(a_{34}\right)$, which shows that $a_{34}=0$.

To finish the rest of the proof we use the following construction: Let

$$
Y=x_{-c}\left(t_{1}\right) x_{c+k}\left(t_{2}\right) x_{2 c+k}\left(t_{3}\right) x_{k}\left(t_{4}\right) x_{3 c+2 k}\left(t_{5}\right),
$$

so that

$$
a(Y)=\left[\begin{array}{cccc}
1 & 0 & t_{1} & t_{3} \\
-t_{1} t_{3}-t_{2} & 1 & t_{4}+t_{1} t_{2} & t_{5}-2 t_{2} t_{3}-t_{1} t_{3}^{2} \\
0 & 0 & 1 & 0 \\
0 & 0 & 0 & 1
\end{array}\right] .
$$

We can make $Y=X$ by taking $t_{1}=a_{13}, t_{3}=a_{14}, t_{2}=-a_{13} a_{14}-a_{21}, t_{4}=a_{23}+$ $a_{13}^{2} a_{14}+a_{21} a_{13}$, and $t_{5}=a_{24}+a_{13} a_{14}^{2}+2 a_{14}\left(-a_{13} a_{14}-a_{21}\right)=a_{24}-a_{13} a_{14}^{2}-2 a_{21} a_{14}$. Because $-c, c+k, 2 c+k, k$, and $3 c+2 k$ are in a system of positive roots, we may apply Lemma (3.3) to conclude that $t_{4}=t_{5}=0$ and that $x_{c}\left(t_{1}\right), x_{c}\left(t_{2}\right), x_{c}\left(t_{3}\right)$ are in $H$. The rest of the proof follows from these relations and (3.2).

(3.5) Lemma. Let $A$ be a commutative ring, and let $H$ be an $E(A)$-normalized subgroup of $G_{2}(A)$ such that $\ell z(H)=0$. Let $J=\ell(H)$ and $M=[E(A), H]$. Then $H=M=E(J, 0)$ and $J_{3}=0$. Furthermore, if $X$ is any element of $H$ and $\tau_{i j}$ denotes the 4,6,i,j minor of $X$, then $\tau_{27}=\tau_{28}=\tau_{38}=\tau_{47}=\tau_{36}=0, \tau_{46}=1$, and $\tau_{26}=\tau_{37}=\tau_{48}$.

Proof. Let $J_{0}$ be the largest ideal of $A$ such that $E\left(J_{0}, 0\right) \subseteq M$, and let $X$ be an arbitrary element of $H$.

Step 1. We show that $\tau_{27}^{2}=\tau_{28}^{2}=\tau_{27} \tau_{28}=\tau_{26} \tau_{28}+\tau_{23} \tau_{78}=0$.

Proof of Step 1. Let $Q=\left[x_{3 c+2 k}(1), X\right]=\left[q_{i j}\right]$, and set

$$
Y=\left[x_{k}\left(-q_{42}\right) x_{3 c+2 k}\left(q_{32}\right), Q\right]=\left[y_{i j}\right] .
$$


Then

$$
a(Y)=\left[\begin{array}{cccc}
1 & 0 & \tau_{12} \tau_{28} & -\tau_{12} \tau_{27} \\
\tau_{18} \tau_{27}-\tau_{17} \tau_{28} & 1 & \tau_{26} \tau_{28}+\tau_{23} \tau_{78} & * \\
0 & 0 & 1+\tau_{27} \tau_{28} & -\tau_{27}^{2} \\
0 & 0 & \tau_{28}^{2} & *
\end{array}\right] .
$$

Applying (3.4) we get immediately that $\tau_{28}^{2}=\tau_{27}^{2}=\tau_{27} \tau_{28}=0$. Lemma (3.4) also gives the relation $y_{23}=-y_{13}^{2} y_{14}-y_{21} y_{13}$. Since $\tau_{28}\left|y_{13}, \tau_{27}\right| y_{14}$, and $y_{21} \in\left(\tau_{27}, \tau_{28}\right)$, it follows that $y_{23}=0$, i.e., that $\tau_{26} \tau_{28}+\tau_{23} \tau_{78}=0$.

Step 2. We show that $\tau_{27}=\tau_{28}=\tau_{38}=0$.

Proof of Step 2. With the notation given above, set $R=\left[x_{3 c+2 k}(1), Q\right], S=$ $\left[x_{3 c+k}(1), R\right]$, and $T=\left[x_{k}(1), R\right]$. Then

$$
a(S)=\left[\begin{array}{cccc}
1 & 0 & \tau_{13} \tau_{28} & 0 \\
-\tau_{18} \tau_{28} & 1 & * & 0 \\
0 & 0 & 1 & 0 \\
0 & 0 & \tau_{28} \tau_{36}-\tau_{38} & 1
\end{array}\right]
$$

Applying (3.4) to $S$ we get that $\tau_{28} \tau_{36}-\tau_{38}=0$. But we know that $\tau_{27} \tau_{28}=0$ from Step 1, and $\tau_{27} \tau_{38}=0$ now follows.

Next, observe that

$$
a(T)=\left[\begin{array}{cccc}
1 & 0 & \tau_{12} \tau_{28} & 0 \\
-\tau_{17} \tau_{28} & 1 & u & v \\
0 & 0 & 1 & 0 \\
0 & 0 & 0 & 1
\end{array}\right]
$$

where $u=-\tau_{28}+\tau_{28} \tau_{26}+\tau_{78} \tau_{23}-\tau_{27} \tau_{38}$ and $v=-\tau_{27}-\tau_{28} \tau_{47}$. But we have just seen that $\tau_{27} \tau_{38}=0$ and Step 1 showed that $\tau_{28} \tau_{26}+\tau_{23} \tau_{78}=0$, so that $u=-\tau_{28}$. Applying (3.4) now shows that $u=v=0$, so we have $\tau_{28}=0$. Since $\tau_{28} \tau_{36}-\tau_{38}=0$, and $v=0$, we now have $\tau_{38}=0$ and $\tau_{27}=0$.

Step 3. We show that $\tau_{47}=\tau_{36}=0, \tau_{26}=\tau_{37}=\tau_{48}$, and that $\tau_{18} \in J_{0}$.

Proof of Step 3. With the conclusions of Step 2 in place, we now have that

$$
a(R)=\left[\begin{array}{cccc}
1 & 0 & 0 & -\tau_{12} \\
\tau_{18} & 1 & 0 & \tau_{48}-\tau_{26} \\
0 & 0 & 1 & 0 \\
0 & 0 & 0 & 1
\end{array}\right]
$$

From (A.11) and (A.10) we have $-\tau_{18} \tau_{12}=\tau_{23} \tau_{78}=\tau_{27} \tau_{38}-\tau_{28} \tau_{37}=0$. Applying (3.4) again shows that $\tau_{48}=\tau_{26}$ and, with (3.2), that $\tau_{18} \in J_{0}$. 
Let $U=\left[x_{k}(1), Q\right]$ so that

$$
a(Q)=\left[\begin{array}{cccc}
1 & 0 & -\tau_{12} & 0 \\
\tau_{17} & 1 & \tau_{37}-\tau_{26} & \tau_{47} \\
0 & 0 & 1 & 0 \\
0 & 0 & 0 & 1
\end{array}\right],
$$

From (A.11) and (A.10) we get that $\tau_{17} \tau_{12}=\tau_{24} \tau_{78}=\tau_{27} \tau_{48}-\tau_{28} \tau_{47}=0$. Then (3.4) shows that $\tau_{26}=\tau_{37}$ and $\tau_{47}=0$.

Now choose an element $\omega \in \mathcal{N}$ which switches 4 and 6 and 3 and 7 . Everything we have done so far applies equally to the conjugate $X^{\omega}$. Since the 4647 minor of $X^{\omega}$ is $\pm \tau_{36}$, we conclude that $\tau_{36}=0$.

Step 4. We show that $\tau_{13}, \tau_{16} \in J_{0}$ and that $\tau_{46}=1$.

Proof of Step 4. Let $V=\left[x_{-k}(1), Q\right]^{\pi}$, where $\pi \in \mathcal{N}$ effects the permutation $(234)(678)$. With the results of Steps 2 and 3 we have that

$$
a(V)=\left[\begin{array}{cccc}
1 & 0 & \tau_{13} & -\tau_{13} \\
\tau_{16}-\tau_{18} & 1 & \tau_{46}-1 & 0 \\
0 & 0 & 1 & 0 \\
0 & 0 & 0 & 1
\end{array}\right] .
$$

By (3.4) and (3.2), $\tau_{16}-\tau_{18} \in J_{0}, \tau_{13} \in J_{0}$, and $\tau_{13}^{3}=3 \tau_{13}=0$. With this and the fact that the $(2,4)$-entry of $V$ is zero, (3.4) allows us to conclude that $\left(\tau_{16}-\tau_{18}\right) \tau_{13}=0$ and hence also that $\tau_{46}=1$. Since $\tau_{18} \in J_{0}$ has already been established, we get that $\tau_{16} \in J_{0}$ as well.

Proof of (3.5). Let $\gamma \in \mathcal{N}$ be an element which switches 4 with 6 and 1 with 5 . Applying Step 4 to $X^{\gamma}$ we get that $\tau_{14}$, the 4616 minor of $X^{\gamma}$, is in $J_{0}$.

We now have that for the matrix $X, \tau_{46}-1=\tau_{36}=\tau_{47}=0$ and $\tau_{13}, \tau_{14}, \tau_{16} \in$ $J_{0}$. Combining these facts with (A.11) we obtain

$$
\begin{aligned}
& a_{41}=a_{44} \tau_{16}-a_{46} \tau_{14}, \\
& a_{43}=a_{44} \tau_{36}-a_{46} \tau_{34}=-a_{46} \tau_{16}, \\
& a_{47}=-a_{44} \tau_{67}+a_{46} \tau_{47}=a_{44} \tau_{14}, \text { and } \\
& a_{48}=-a_{44} \tau_{68}+a_{46} \tau_{14}=-a_{44} \tau_{13}+a_{46} \tau_{14} .
\end{aligned}
$$

This shows that $a_{41}, a_{43}, a_{47}, a_{48}$ are in $J_{0}$.

Applying this last result to conjugates of $X$ and $X^{-1}$ by elements of $\mathcal{N}$ and using (A.4), we see that $a_{i j} \in J_{0}$ for all $i \neq j$ except when $\{i, j\}=\{1,5\}$. Using (A.6) and computing the $(2,2)$-entry of $b(X) c(X)^{T}$, we get

$$
1-a_{11}=-a_{21} a_{61}+a_{26} a_{62}+a_{27} a_{63}+a_{28} a_{64} .
$$

It follows that $a_{15}=a_{51}=1-a_{11}$ all belong to $J_{0}$.

Now the $(3,1)$-entry of $X^{x_{c}(1)}$ is in $J_{0}$. This entry is $2 a_{11}-1+a_{31}+a_{71}-2 a_{13}-$ $a_{33}-a_{73}$. Thus, $a_{33}-1 \in J_{0}$. Applying this result to conjugates of $X$ by elements of $\mathcal{N}$, we finally have that $\ell(X) \subseteq J_{0}$. Since $X$ was an arbitrary element of $H$, we conclude that $J_{0}=J$. Because we now have shown that $E(J, 0) \subseteq M,(3.2)$ proves that $J_{3}=0$. It follows from $(2.7)$ that $G(J)=E(J)$. 
From our definition, $E(J)=E(J, 0) E(0, J)$. So an arbitrary element $X$ in $H$ can be written $X=X_{1} X_{2}$, where $X_{1} \in E(J, 0)$ and $X_{2} \in E(0, J)$. But $E(J, 0) \subseteq M \subseteq$ $H$, so that $X_{1} \in H$ and hence $X_{2} \in H \cap E(0, J) \subseteq H \cap S L(3, A)$. Our hypothesis that $\ell z(H)=0$ says that $X_{2}$ is a scalar in $S L(3, A)$, i.e., that $a\left(X_{2}\right)$ has the form $\left[\begin{array}{ll}1 & 0 \\ 0 & \lambda I\end{array}\right]$, where $\lambda-1 \in J$. Then $X_{2}=\sigma\left(\left[\begin{array}{cc}\lambda & 0 \\ 0 & \lambda^{2}\end{array}\right]\right)$, and it is easily seen that this matrix is in $E(J, 0)$. Thus, $X \in E(J, 0)$, and we have shown that $H=E(J, 0)$. This completes the proof of the lemma.

(3.6) Main Theorem. Let $A$ be a commutative ring and let $H$ be an $E(A)$ normalized subgroup of $G_{2}(A)$. Let $J=\ell(H)$ and $J^{\prime}=\ell z(H)$. Then $J_{3} \subseteq J^{\prime}$ and

$$
C\left(J, J^{\prime}\right) \subseteq H \subseteq G\left(J, J^{\prime}\right)
$$

Moreover, $[E(A), H]=C\left(J, J^{\prime}\right)$.

Proof. Using overbars to simultaneously denote passage from $A$ to $A / J^{\prime}$ or from $G_{2}(A)$ to $G_{2}\left(A / J^{\prime}\right)$, the first thing we need to establish is that the $A_{2}$-level of $\bar{H}$ is 0. Suppose that, in fact, $\ell z(\bar{H})=\bar{J}^{*}$, where $J^{*}$ is an ideal of $A$ containing $J^{\prime}$. Then in $G_{2}(\bar{A})$ we have that $\bar{H} \cap S L(3, \bar{A})$ is an $E(3, \bar{A})$-normalized subgroup of $S L(3, \bar{A})$ of level $\bar{J}^{*}$, so it contains $E\left(0, \bar{J}^{*}\right)$. Thus, $C\left(0, J^{*}\right) \subseteq H G\left(J^{\prime}\right)$. Since, as previously remarked, $C\left(J^{\prime}\right) \subseteq H$, the reduction lemma, Lemma (3.1), now shows that $C\left(0, J^{*}\right) \subseteq H$. But this means that $C\left(0, J^{*}\right) \subseteq H \cap S L(3, A)$, from which we get $J^{*} \subseteq \ell z(H)=J^{\prime}$. Thus, $\ell z(\bar{H})=\bar{J}^{*}=0$.

Now we may apply (3.5) to $\bar{H}$. This tells us that $\bar{J}_{3}=0$ and $\bar{H}=E(\bar{J}, 0)$. We immediately conclude that $J_{3} \subseteq J^{\prime}$. Moreover, $\overline{C\left(J, J^{\prime}\right)}=C(\bar{J}, 0) \subseteq E(\bar{J}, 0)=\bar{H}$, which says that $C\left(J, J^{\prime}\right) \subseteq H G\left(J^{\prime}\right)$. Again, from (3.1) it follows that $C\left(J, J^{\prime}\right) \subseteq H$. The fact that $\bar{H}=E(\bar{J}, 0)=\overline{E(J, 0)}$ also says that $H \subseteq E(J, 0) G\left(J^{\prime}\right)=G\left(J, J^{\prime}\right)$. This proves that $C\left(J, J^{\prime}\right) \subseteq H \subseteq G\left(J, J^{\prime}\right)$. Finally, we have

$$
\begin{aligned}
C\left(J, J^{\prime}\right) & =\left[E(A), C\left(J, J^{\prime}\right)\right] \subseteq[E(A), H] \subseteq\left[E(A), G\left(J, J^{\prime}\right)\right] \\
& =C\left(J, J^{\prime}\right),
\end{aligned}
$$

completing the proof of the theorem.

Notice that the main theorem characterizes the $E(A)$-normalized subgroups of $G_{2}(A)$ as those falling in a strip between $C\left(J, J^{\prime}\right)$ and $G\left(J, J^{\prime}\right)$, where $J, J^{\prime}$ are ideals such that $J_{3} \subseteq J^{\prime} \subseteq J$. Are all such pairs of ideal possible? The answer is yes. In fact, the groups $C\left(J, J^{\prime}\right)$ and $G\left(J, J^{\prime}\right)$ are uniquely determined by these pairs. These facts are part of the next lemma.

(3.7) Lemma. Let $J$ and $J^{\prime}$ be ideals of $A$ such that $J_{3} \subseteq J^{\prime} \subseteq J$. Then

(i) $\ell\left(C\left(J, J^{\prime}\right)\right)=\ell\left(G\left(J, J^{\prime}\right)\right)=J$;

(ii) $\ell z\left(C\left(J, J^{\prime}\right)\right)=\ell z\left(G\left(J, J^{\prime}\right)\right)=J^{\prime}$.

Consequently, there are bijections between the set of such pairs of ideals and the sets of subgroups of $G_{2}(A)$ of the form $C\left(J, J^{\prime}\right)$ and $G\left(J, J^{\prime}\right)$, respectively. Moreover, $G\left(J, J^{\prime}\right)$ is the largest $E(A)$-normalized subgroup $H$ of $G_{2}(A)$ such that $\ell(H) \subseteq J$ and $\ell z(H) \subseteq J^{\prime}$.

Proof. Item (i) is clear. To prove (ii), note that $J^{\prime} \subseteq \ell z\left(C\left(J, J^{\prime}\right)\right) \subseteq \ell z\left(G\left(J, J^{\prime}\right)\right)$, so we only need to argue that $\ell z\left(G\left(J, J^{\prime}\right)\right) \subseteq J^{\prime}$. It suffices to do this modulo $J^{\prime}$, so we may assume that $J^{\prime}=0$, and hence that $J_{3}=0$. Now $G\left(J, J^{\prime}\right)=G(J, 0)=E(J, 0)$, 
so that $\ell z\left(G\left(J, J^{\prime}\right)\right)=\ell z(E(J, 0))=0$, by (1.15). This proves (ii). The assertion about bijections is clear and the characterization of $G\left(J, J^{\prime}\right)$ now follows from the main theorem.

We now have the following corollary:

(3.8) Corollary. Let $A$ be a commutative ring, and let $K$ be an $E(A)$-normalized subgroup of $G_{2}(A)$ such that $[E(A), K]=C\left(J, J^{\prime}\right)$, where $J, J^{\prime}$ are ideals of $A$ such that $J_{3} \subseteq J^{\prime} \subseteq J$. Then $K \subseteq G\left(J, J^{\prime}\right)$.

Proof. From the main theorem and (3.7) it is clear that $\ell(K)=J$ and $\ell z(K)=J^{\prime}$, from which it follows that $K \subseteq G\left(J, J^{\prime}\right)$.

We close this section by proving the normality of the subgroups $C\left(J, J^{\prime}\right)$ and $G\left(J, J^{\prime}\right)$. A key tool is Vaserstein's theorem [16] that $E(J)$ is normal in $G_{2}(A)$ for each ideal $J$ of $A$. Since $C(J)=[E(A), E(J)]$ by (1.7), it follows from Vaserstein's theorem that $C(J)$ is also normal in $G_{2}(A)$.

(3.9) Lemma. Let $A$ be a commutative ring, and let $N_{1}, N_{2}$ be $G_{2}(A)$-conjugate, $E(A)$-normalized subgroups of $G_{2}(A)$. Then $\ell z\left(N_{1}\right)=\ell z\left(N_{2}\right)$.

Proof. Let $J_{i}=\ell z\left(N_{i}\right), i=1,2$. Then $C\left(J_{i}\right) \subseteq N_{i}, i=1,2$. But we have just seen that $C\left(J_{i}\right)$ is normal in $G_{2}(A)$, so the fact that $N_{1}, N_{2}$ are conjugates implies that $C\left(J_{1}\right) \subseteq N_{2}$ and $C\left(J_{2}\right) \subseteq N_{1}$ also hold. It follows that $J_{1}=J_{2}$.

(3.10) Theorem. Let $A$ be a commutative ring and let $J, J^{\prime}$ be ideals of $A$ such that $J_{3} \subseteq J^{\prime} \subseteq J$. Then $G\left(J, J^{\prime}\right)$ and $C\left(J, J^{\prime}\right)$ are normal subgroups of $G_{2}(A)$.

Proof. Let $\gamma \in G_{2}(A)$, and let $N=G\left(J, J^{\prime}\right)^{\gamma}$. Then $N$ is $E(A)$-normalized and $\ell(N)=J$ and $\ell z(N)=J^{\prime}$ by (3.7) and (3.9). The main theorem now shows that $N \subseteq G\left(J, J^{\prime}\right)$. This proves that $G\left(J, J^{\prime}\right)$ is normal in $G_{2}(A)$. Since $C\left(J, J^{\prime}\right)=$ $\left[E(A), G\left(J, J^{\prime}\right)\right]$ by $(1.21)$, it, too, is normal.

\section{Determination of the $A_{2}$-Level}

The purpose of this section is to show that, for a given matrix $X$ in $G_{2}(A)$, the $A_{2}$-level of $X$ can be computed from its entries in a very explicit way. Recall that the $A_{2}$-level of $X, \ell z(X)$, is defined to be the $A_{2}$-level of the $E(A)$-normalized subgroup $N(X)$ generated by $X$, i.e., $\ell z(X)=\ell(N(X) \cap S L(3, A))$. For this reason we will be concerned with level ideals of elements of $S L(3, A)$. It will turn out that we can best examine these elements by passing to $P S L(3, A)$. We note that if $Y \in S L(3, A)$ and $\bar{Y}$ is its image in $\operatorname{PSL}(3, A)$, then $\bar{Y}$ is the coset $Y L(3, A ; 0)$. Since elements of this coset are unit multiples of $Y$, they all have the same level ideal in $A, \ell(Y)$, and so we may unambiguously denote this ideal by $\ell(\bar{Y})$. In other words, to compute $\ell(Y)$ it suffices to identify the $L(3, A ; 0)$-coset to which $Y$ belongs and compute the level ideal of any representative of that coset. In this way, we may compute level ideals by doing computations in $\operatorname{PSL}(3, A)$.

Now let $J$ be an ideal of $A$. We are going to construct a surjective group homomorphism $\zeta_{J}: G(J) \rightarrow P S L\left(3, A / J_{3} ; J / J_{3}\right)$, which we will use in the sequel. To do this, we begin by recalling that $(2.9)$ showed that $E(3, A ; J) G\left(J, J_{3}\right)=G(J)$; hence $G(J)=S L(3, A ; J) G\left(J, J_{3}\right)$. From (3.7) it is clear that

$$
S L(3, A ; J) \cap G\left(J, J_{3}\right)=S L(3, A ; J) \cap L\left(3, A ; J_{3}\right) .
$$


There is therefore a natural isomorphism

$$
\frac{G(J)}{G\left(J, J_{3}\right)} \longrightarrow \frac{S L(3, A ; J)}{S L(3, A ; J) \cap L\left(3, A ; J_{3}\right)} .
$$

The latter group is the image of $S L(3, A ; J)$ in $P S L\left(3, A / J_{3}\right)$, namely $P S L\left(3, A / J_{3} ; J / J_{3}\right)$. Composing this map with the canonical surjection of $G(J)$ onto the first group, we obtain the desired homomorphism $\zeta_{J}$. Note that $G\left(J, J_{3}\right)=$ $\operatorname{ker}\left(\zeta_{J}\right)$.

[For reference, recall that $P S L(3, A)=S L(3, A) / L(3, A ; 0)$. For an ideal $J$ of $A$, we write $P S L(3, A ; J)$ to denote the image of $S L(3, A ; J)$ in $P S L(3, A)$.]

The first lemma is a refinement of (2.9).

(4.1) Lemma. Let $J$ be an ideal of a commutative ring $A$.

(i) If $J^{\prime}$ is an ideal such that $J_{3} \subseteq J^{\prime} \subseteq J$, then

$$
G\left(J, J^{\prime}\right)=G\left(J, J_{3}\right) E\left(3, A ; J^{\prime}\right) .
$$

(ii) If $N$ is an $E(A)$-normalized subgroup of $G_{2}(A)$ such that $G\left(J, J_{3}\right) \subseteq N \subseteq$ $G(J)$ and $\ell z(N)=J^{\prime}$, then $N=G\left(J, J^{\prime}\right)$.

Proof. First we observe that (i) implies (ii), for the hypotheses in (ii) imply that $J_{3} \subseteq J^{\prime} \subseteq J$ and the Main Theorem shows that $N \subseteq G\left(J, J^{\prime}\right)$. On the other hand, $\ell z(N)=J^{\prime}$ implies that $N \cap S L(3, A) \supseteq E\left(3, A ; J^{\prime}\right)$; hence $G\left(J, J^{\prime}\right) E\left(3, A ; J^{\prime}\right) \subseteq N$. Thus, it suffices to prove (i).

Now $G\left(J, J_{3}\right)=G\left(J_{3}\right) E(J, 0)$, so (i) is the assertion that

$$
G\left(J, J^{\prime}\right)=G\left(J_{3}\right) E(J, 0) E\left(3, A ; J^{\prime}\right) .
$$

Since the right-hand side of this equation consists of products of elementaries modulo $G\left(J_{3}\right)$, it suffices to prove (i) under the hypothesis that $J_{3}=0$.

Since $J_{3}=0$, we have that $S R_{2}(A, J)$ and $S R_{2}\left(A, J^{\prime}\right)$ hold, so $S L\left(3, A ; J^{\prime}\right)=$ $E\left(3, A ; J^{\prime}\right)$ and $S L(3, A ; J)=E(3, A ; J)$. Also, $G\left(J, J_{3}\right)=E(J, 0) G\left(J_{3}\right)=E(J, 0)$. Now

$$
G\left(J, J_{3}\right) \subseteq G\left(J, J^{\prime}\right) \subseteq G(J)=G\left(J, J_{3}\right) E(3, A ; J),
$$

by (2.9). Thus,

$$
G\left(J, J^{\prime}\right)=G\left(J, J_{3}\right)\left[G\left(J, J^{\prime}\right) \cap S L(3, A ; J)\right] .
$$

But $G\left(J, J^{\prime}\right) \cap S L(3, A ; J)=S L(3, A ; J) \cap L\left(3, A ; J^{\prime}\right)$. Consider a matrix $X$ in this last intersection, so $a(X)=\left[\begin{array}{ll}1 & 0 \\ 0 & Y\end{array}\right]$, where

$$
Y=\left[\begin{array}{ccc}
y_{11} & & * \\
& y_{22} & \\
* & & y_{33}
\end{array}\right],
$$

and $y_{11}=1+j, y_{22}=y_{11}+k, y_{33}=y_{11}+\ell$, where $j \in J$ and $k, \ell \in J^{\prime}$. Then $y_{11}^{3}=1$ because $J_{3}=0$. This means that $Y=Y_{1} Y_{2}$, where

$$
Y_{1}=\left[\begin{array}{ccc}
y_{11} & 0 & 0 \\
0 & y_{11} & 0 \\
0 & 0 & y_{11}
\end{array}\right] \text {, }
$$


and $Y_{2} \equiv I \bmod J^{\prime}$. Let $X_{1}, X_{2} \in G_{2}(A)$ be the elements such that $a\left(X_{1}\right)=\left[\begin{array}{cc}1 & 0 \\ 0 & Y_{1}\end{array}\right]$ and $a\left(X_{2}\right)=\left[\begin{array}{cc}1 & 0 \\ 0 & Y_{2}\end{array}\right]$. Then $X_{2} \in S L\left(3, A ; J^{\prime}\right)$ and, as noted in the proof of (3.5), $X_{1} \in E(J, 0)=G\left(J, J_{3}\right)$. It follows that

$$
G\left(J, J^{\prime}\right) \subseteq G\left(J, J_{3}\right) S L\left(3, A ; J^{\prime}\right)=G\left(J, J_{3}\right) E\left(3, A ; J^{\prime}\right),
$$

and this completes the proof.

Note that it follows from (4.1) that if $J_{3} \subseteq J^{\prime} \subseteq J$, then

$$
\zeta_{J}\left(G\left(J, J^{\prime}\right)\right)=\operatorname{PSL}\left(3, A / J_{3} ; J^{\prime} / J_{3}\right) .
$$

(4.2) Lemma. Let $J$ be an ideal of a commutative ring $A$ such that $J_{3}=0$. If $S$ is a subset of $G_{2}(A)$ and $\ell(S)=J$, then $\ell z(S)=\ell\left(\zeta_{J}(S)\right)$.

Proof. Let $N$ be the $E(A)$-normalized subgroup of $G_{2}(A)$ generated by $S$. Then $\ell(N)=J$ and hence $C(J, 0) \subseteq N$ by (3.6). Since $3 J=0$, it follows that $E(J, 0) \subseteq N$ by (1.5). But $E(J, 0)=G\left(J, J_{3}\right)=\operatorname{ker}\left(\zeta_{J}\right)$, so we have that $G\left(J, J_{3}\right) \subseteq N$. Setting $J^{\prime}=\ell z(N),(4.1)$ tells us that $N=G\left(J, J^{\prime}\right)$ and hence that $\zeta_{J}(N)=P S L\left(3, A ; J^{\prime}\right)$. Thus, $\ell z(N)=\ell\left(\zeta_{J}(N)\right)$.

Let $J^{\prime \prime}=\ell\left(\zeta_{J}(S)\right)$. Then $J^{\prime \prime} \subseteq J^{\prime}$. By definition, $\ell_{z}(S)=\ell z(N)=J^{\prime}$, so it only remains to show that $J^{\prime} \subseteq J^{\prime \prime}$. This is equivalent to showing that $G\left(J, J^{\prime}\right) \subseteq$ $G\left(J, J^{\prime \prime}\right)$ and therefore, in our case, to showing that $S \subseteq G\left(J, J^{\prime \prime}\right)$. So let $X \in S$. We know that $\zeta_{J}(X) \in P S L(3, A ; J)$ and $\ell\left(\zeta_{J}(X)\right) \subseteq J^{\prime \prime}$. Thus, $\zeta_{J}(X)=Y L(3, A ; 0)$, where $Y \in S L(3, A ; J) \cap L\left(3, A ; J^{\prime \prime}\right)$. As in the proof of $(4.1)$, such an element $Y$ can be written as $Y=Y_{1} Y_{2}$, where $Y_{1} \in L(3, A ; 0)$ and $Y_{2} \in S L\left(3, A ; J^{\prime \prime}\right)$. It follows that

$$
\zeta_{J}(X)=Y_{2} L(3, A ; 0) \in P S L\left(3, A ; J^{\prime \prime}\right)=\zeta_{J}\left(G\left(J, J^{\prime \prime}\right)\right) .
$$

Since $\operatorname{ker}\left(\zeta_{J}\right)=E(J, 0) \subseteq G\left(J, J^{\prime \prime}\right)$, this proves that $X \in G\left(J, J^{\prime \prime}\right)$ and completes the proof of the lemma.

The next lemma shows, in essence, that $\ell z(S)$ can be computed modulo $\ell(S)_{3}$. Put together with (4.2) this effectively allows us to calculate $A_{2}$-levels as level ideals in $P S L\left(3, A / \ell(S)_{3}\right)$. In order to state the next result, let $\nu: A \rightarrow A / J_{3}$ be the canonical ring homomorphism and use overbars to denote images under $\nu$. For example, $\bar{A}$ denotes $A / J_{3}$ and if $N \subseteq G_{2}(A)$, then $\bar{N}$ is its image in $G_{2}(\bar{A})$, or, equivalently, its image modulo $G\left(J_{3}\right)$.

(4.3) Lemma. Let $A$ be a commutative ring, and let $S$ be a subset of $G_{2}(A)$. Set $J=\ell(S)$, and let $\nu: A \rightarrow A / J_{3}$ be the canonical map. Then $\ell z(S)=\nu^{-1}(\ell z(\bar{S}))=$ $\nu^{-1}\left(\ell\left(\zeta_{J}(S)\right)\right)$.

Proof. The second equality follows from (4.2), so we only need to verify the first one. Let $N$ be the $E(A)$-normalized subgroup of $G_{2}(A)$ generated by $S$. Then $\bar{N}$ is the $E(\bar{A})$-normalized subgroup of $G_{2}(\bar{A})$ generated by $\bar{S}$. Since $J_{3} \subseteq \ell(S)=\ell(N)$ by (3.6), it suffices to show that $\overline{\ell z(N)}=\ell z(\bar{N})$.

It is clear that $\overline{N \cap S L(3, A)} \subseteq \bar{N} \cap S L(3, \bar{A})$ and hence that $\overline{\ell z(N)} \subseteq \ell z(\bar{N})$. On the other hand, from (3.6) we have that $N \subseteq G\left(J, J^{\prime}\right)$, where $J^{\prime}=\ell z(N)$, so that $\ell z(\bar{N}) \subseteq \ell z\left(\overline{G\left(J, J^{\prime}\right)}\right)$. Thus, it suffices to prove that $\ell z(\bar{N}) \subseteq \overline{\ell z(N)}$ in the case that $N=G\left(J, J^{\prime}\right)$. This means that we may proceed under the assumption that $N \supseteq G\left(J_{3}\right)=\operatorname{ker}\left(G_{2}(A) \rightarrow G_{2}(\bar{A})\right)$. Since $\ell(\bar{N})=\bar{J}$,

$$
\bar{N} \cap S L(3, \bar{A})=\bar{N} \cap S L(3, \bar{A} ; \bar{J})=\bar{N} \cap E(3, \bar{A} ; \bar{J})=\bar{N} \cap \overline{E(3, A ; J)} .
$$


Because $N \supseteq G\left(J_{3}\right)$, however, we get that $\bar{N} \cap \overline{E(3, A ; J)}=\overline{N \cap E(3, A ; J)}$ from which it follows that $\ell z(\bar{N}) \subseteq \overline{\ell z(N)}$. This completes the proof of the lemma.

From Lemma (4.3) it should now be clear that for an $X \in G_{2}(A), \ell z(X)$ may be computed as follows: write $X=X_{1} X_{2}$ with $X_{1} \in G\left(\ell(X), \ell(X)_{3}\right)$ and $X_{2} \in$ $S L(3, A)$, and then $\ell z(X)=\ell\left(X_{2}\right)+\ell(X)_{3}$.

We adopt the following notation: For matrices $X \in G_{2}(A)$, we let $Y(X)$ be the $3 \times 3$ block such that $a(X)=\left[\begin{array}{l}* \\ * Y(X)\end{array}\right]$. If $X \in S L(3, A)$, we use braces $\{Y(X)\}$ to denote the image of this matrix in $P S L(3, A)$ (or $P S L(3, \bar{A})$, if appropriate).

(4.4) Lemma. Let $J$ be an ideal in a commutative ring $A$ such that $J_{3}=0$. Let

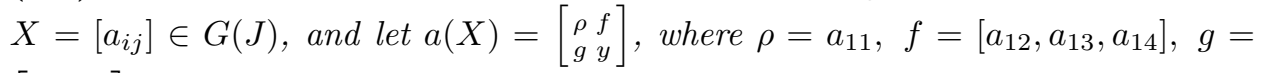
$\left[\begin{array}{c}a_{21} \\ a_{31} \\ a_{41}\end{array}\right], y=Y(X)$. Set $u=\left[a_{61}, a_{71}, a_{81}\right]$. Then

$$
\zeta_{J}(X)=\left\{Y\left(L(u)^{T}\right) Y(L(g))(y+g f)\right\} .
$$

Proof. We apply (2.8). Since $S\left(f y^{-1}\right)$ and $S(g)$ are in $E(J, 0)=\operatorname{ker} \zeta_{J}$,

$$
\zeta_{J}(X)=\left\{Y\left(L\left(f y^{-1}\right)^{T}\right) Y(L(g))\left(\rho I-g f y^{-1}\right)^{-1} y\right\} .
$$

Now $f y^{-1} g=\rho-1$ and $f y^{-1}=\rho^{-1} u$, by (A.6). But $\rho^{3}=1$ and the matrix $L\left(t_{1}, t_{2}, t_{3}\right)$ is defined by polynomials in $t_{1}, t_{2}, t_{3}$ in which every monomial has degree divisible by 3 , so that $L\left(f y^{-1}\right)=L(u)$. One checks that $\left(\rho I-g f y^{-1}\right)^{-1}=$ $\rho^{-1}\left(I+g f y^{-1}\right)$ by multiplying. Then $\left\{\rho^{-1}\left(I+g f y^{-1}\right) y\right\}=\{y+g f\}$, and the proof is complete.

We note that if $J$ is an ideal of $A$ which is locally principal, then $J^{3} \subseteq J_{3}$ which makes $L(u)=L(g)=I$ above. This holds, for example, if $J$ is principal or if $J$ is any ideal in a Dedekind ring. In these special cases, Lemma (4.4) becomes the following theorem.

(4.6) Theorem. Let $J$ be an ideal of a commutative ring $A$ such that $J^{3} \subseteq J_{3}$, and let $X \in G(J)$, with $a(X)=\left[\begin{array}{ll}\rho & f \\ g & y\end{array}\right]$. Then

$$
\zeta_{J}(X)=\{y+g f\}
$$

Putting everything together gives a nice way to compute $\ell z$ in these cases:

(4.7) Corollary. Let $A$ be a commutative ring, and let $X \in G_{2}(A)$, with $a(X)=$ $\left[\begin{array}{ll}\rho & f \\ g & y\end{array}\right]$. If $\ell(X)^{3} \subseteq \ell(X)_{3}$, then $\ell z(X)=\ell(y+g f)+\ell(X)_{3}$.

The computation of $\ell z(X)$ in the general case requires more work. It is clear that by using (4.5) with $J=\ell(X)$ we could obtain 8 polynomials in the entries of $X$ which generate $\ell z(X)$ modulo $\ell(X)_{3}$. These polynomials seem to us to be too cumbersome to present here. Instead, we proceed with an alternate computation of $\ell z(X)$ in terms of $2 \times 2$ minors of $X$.

For $X$ an arbitrary element of $G_{2}(A)$, let $m_{0}(X)$ be the ideal

$$
m_{0}(X)=\left(\tau_{27}, \tau_{28}, \tau_{36}, \tau_{38}, \tau_{46}-1, \tau_{47}, \tau_{26}-\tau_{37}\right)+\ell(X)_{3},
$$

where $\tau_{i j}$ is the $4,6, i, j$ minor of $X$. Let $\pi=(234)(678)$ and set $m(X)=m_{0}(X)+$ $m_{0}\left(X^{\pi}\right)+m_{0}\left(X^{\pi^{2}}\right)$. 
For any permutation $\sigma$ in $S L(8, \mathbf{Z})$ the $i, j, k, \ell$ minor of $X^{\sigma}$ is the

$$
\sigma^{-1}(i), \sigma^{-1}(j), \sigma^{-1}(k), \sigma^{-1}(\ell)
$$

minor of $X$, so $m(X)$ can be expressed in terms of 24 minors of $X$.

(4.8) Theorem. Let $A$ be a commutative ring, and let $X$ be an element of $G_{2}(A)$. Then $m(X)$ is the $A_{2}$-level of $X$.

Proof. In (3.5) we have shown that $m_{0}(X)$ is contained in $\ell z(X)$. This makes it clear that $m(X) \subseteq \ell z(X)$. It remains to prove the opposite inclusion. In what follows, we make free use of (A.10) and (A.11). We set $J=\ell(X)$.

Claim 1. The $A_{2}$-level of $Q=\left[x_{3 c+2 k}(1), X\right]$ is contained in $m_{0}(X)$. 1.

To prove this we go modulo $m_{0}(X)$, so that $m_{0}(X)=0$, and show that $\zeta_{J}(Q)=$

Since $J_{3}=0$ and $\tau_{26}=\tau_{37}$, we have

$$
\begin{aligned}
\tau_{26} & =\tau_{48}=\tau_{37}=\tau_{37} \tau_{46}=\tau_{34} \tau_{76}-\tau_{36} \tau_{74} \\
& =-\tau_{34} \tau_{67}=\tau_{14} \tau_{16} .
\end{aligned}
$$

Now $\tau_{13}=\tau_{13} \tau_{46}=\tau_{14} \tau_{36}-\tau_{16} \tau_{34}=-\tau_{16}^{2}$. Also, $\tau_{17}=\tau_{17} \tau_{46}=\tau_{14} \tau_{76}-\tau_{16} \tau_{74}=$ $-\tau_{14} \tau_{67}=\tau_{14}^{2}$, so

$$
\tau_{12}=\tau_{12} \tau_{46}=\tau_{14} \tau_{26}-\tau_{16} \tau_{24}=\tau_{14} \tau_{26}+\tau_{16} \tau_{17}=-\tau_{14}^{2} \tau_{16}
$$

Finally,

$$
\tau_{18}=\tau_{18} \tau_{46}=\tau_{14} \tau_{86}-\tau_{16} \tau_{84}=-\tau_{14} \tau_{13}+\tau_{16} \tau_{48}=-\tau_{14} \tau_{16}^{2} .
$$

Computing $\zeta_{J}(Q)$ using $m_{0}(X)=0$ and the relations just derived we get

$$
\zeta_{J}(Q)=\left\{E_{13}\left(\tau_{16}^{2} \tau_{14}^{2}\right) E_{13}\left(\tau_{16}^{2} \tau_{14}^{2}\right)\left[\begin{array}{ccc}
\eta & 0 & \tau_{16}^{2} \tau_{14}^{2} \\
0 & \eta & 0 \\
0 & 0 & \eta
\end{array}\right]\right\},
$$

where $\eta=1+\tau_{16} \tau_{14}+\tau_{16}^{2} \tau_{14}^{2}$. It follows that $\zeta_{J}(Q)=1$ and hence that $\ell z(Q) \subseteq$ $m_{0}(X)$, by (4.2). This proves Claim 1 .

Claim 2. The $A_{2}$-levels of $\left[x_{-k}(1), X\right]$ and $\left[x_{-3 c-k}(1), X\right]$ are contained in $m(X)$.

We have $\left[x_{-3 c-k}(1), X\right]^{\pi}=\left[x_{3 c+2 k}(1), X^{\pi}\right]$ and $\left[x_{-k}(1), X\right]^{\pi^{2}}=\left[x_{3 c+2 k}(1), X^{\pi^{2}}\right]$. Applying Claim 1 to $X^{\pi}$ and $X^{\pi^{2}}$ proves Claim 2.

To finish the proof of the theorem, we may assume that $m(X)=0$ and show that $\zeta_{J}(X)=1$. We have seen in Claims 1 and 2 that if

$$
\phi \in\{-k,-3 c-k, 3 c+2 k\},
$$

then $\zeta_{J}\left(\left[x_{\phi}(1), X\right]\right)=1$. Thus,

$$
1=\zeta_{J}\left(\left(X^{-1}\right)^{x_{\phi}(1)}\right) \zeta_{J}(X)=\left[\left\{Y\left(x_{\phi}(1)\right)\right\}, \zeta_{J}(X)\right],
$$

i.e., that $\left\{Y\left(x_{\phi}(1)\right)\right\}$ centralizes $\zeta_{J}(X)$ in $P S L(3, A)$. But

$$
\left\{Y\left(x_{\phi}(1)\right) \mid \phi \in\{-k,-3 c-k, 3 c+2 k\}\right\}
$$

generates $E(3, B)$, where $B$ is the prime subring of $A$. Now Lemma (A.16) shows that $\zeta_{J}(X)=1$, and the proof of the theorem is complete. 


\section{APPENDIX}

Here we develop our particular representation of $G_{2}(A)$ as a subgroup of $G L(8, A)$ by using the fact that $G_{2}(A)$ can be viewed as the automorphism group of the split Cayley algebra over $A$.

Let $A$ be a commutative ring with identity. We shall denote by $\mathcal{O}(A)$ the split Cayley algebra over $A$ (also called the split octonions over $A$ ). Our development is taken from Jacobson [13], with extra detail provided by J. Faulkner. The algebra $\mathcal{O}(A)$ is the set of all " $2 \times 2$ matrices" $\left(\begin{array}{ll}\alpha & a \\ b & \beta\end{array}\right)$ such that $\alpha, \beta \in A$, and

$$
a=\left[\begin{array}{l}
a_{1} \\
a_{2} \\
a_{3}
\end{array}\right], b=\left[\begin{array}{l}
b_{1} \\
b_{2} \\
b_{3}
\end{array}\right]
$$

are elements (vectors) in the rank 3 free $A$-module $A^{3}$. Addition and scalar multiplication in $\mathcal{O}(A)$ are defined component-wise, so that $\mathcal{O}(A)$ is a free $A$-module of rank 8. The multiplication in $\mathcal{O}(A)$ is defined by the Zorn product

$$
\left(\begin{array}{ll}
\alpha & a \\
b & \beta
\end{array}\right)\left(\begin{array}{ll}
\gamma & c \\
d & \delta
\end{array}\right)=\left(\begin{array}{cc}
\alpha \gamma-a \cdot d & \alpha c+\delta a+b \times d \\
\gamma b+\beta d+a \times c & \beta \delta-b \cdot c
\end{array}\right)
$$

where · and $\times$ denote the usual vector "dot product" and "cross product," respectively, in $A^{3}$. The $\operatorname{ring} A$ is naturally identified with a subalgebra of $\mathcal{O}(A)$ via the embedding $\alpha \mapsto\left(\begin{array}{ll}\alpha & 0 \\ 0 & \alpha\end{array}\right)$, for all $\alpha \in A$. This makes $\mathcal{O}(A)$ a non-associative $A$-algebra. (A weak form of associativity does hold in $\mathcal{O}(A)$ : for all $x, y \in \mathcal{O}(A)$, $x^{2} y=x(x y)$ and $x y^{2}=(x y) y$, i.e, $\mathcal{O}(A)$ is an alternative algebra.)

Involution, trace, norm. There is a natural involution, $x \mapsto \bar{x}$, on $\mathcal{O}(A)$ defined by $\left(\begin{array}{ll}\alpha & a \\ b & \beta\end{array}\right) \mapsto\left(\begin{array}{cc}\beta & -a \\ -b & \alpha\end{array}\right)$. Using this involution one defines the trace and norm of an element $x \in \mathcal{O}(A)$ by $\operatorname{Tr}(x)=x+\bar{x}$ and $N(x)=x \bar{x}$, respectively. It is easily checked that these are elements of $A$. In fact, if $x=\left(\begin{array}{ll}\alpha & a \\ b & \beta\end{array}\right)$, then $\operatorname{Tr}(x)=\alpha+\beta$ and $N(x)=\alpha \beta+a \cdot b$. Note that $\operatorname{Tr}$ is an $A$-linear map and $N(x y)=N(x) N(y)$.

The trace and norm are, in turn, used to define the bilinear form $N(x, y)=$ $N(x+y)-N(x)-N(y)=\operatorname{Tr}(x \bar{y})$ and the trilinear form $T(x, y, z)=N(x y, z)=$ $\operatorname{Tr}((x y) \bar{z})$.

Letting $\operatorname{Aut}(\mathcal{O}(A))$ be the group of involution-preserving $A$-algebra automorphisms of $\mathcal{O}(A)$, we have $G_{2}(A)=\operatorname{Aut}(\mathcal{O}(A))$. Since the trace, the norm, the bilinear form, and the trilinear form on $\mathcal{O}(A)$ are all defined using the involution, the elements of $G_{2}(A)$ preserve all of these. Conversely, it can be shown that any $A$ module automorphism of $\mathcal{O}(A)$ which preserves $N(x, y)$ and $T(x, y, z)$ is an element of $G_{2}(A)$.

Ordered bases. Let $A^{3}$ have ordered basis $e_{2}, e_{3}, e_{4}$. We fix an ordered basis

$$
\mathcal{B}=\left\{u_{1}, u_{2}, u_{3}, u_{4}, u_{5}, u_{6}, u_{7}, u_{8}\right\}
$$

for $\mathcal{O}(A)$ as follows: set $u_{1}=\left(\begin{array}{ll}1 & 0 \\ 0 & 0\end{array}\right), u_{5}=\left(\begin{array}{ll}0 & 0 \\ 0 & 1\end{array}\right), u_{i}=\left(\begin{array}{ll}0 & e_{i} \\ 0 & 0\end{array}\right)$ for $i=2,3,4$, and $u_{j}=\left(\begin{array}{cc}0 & 0 \\ e_{j-4} & 0\end{array}\right)$ for $j=6,7,8$. 
Using this basis, an element

$$
x=\left(\begin{array}{cc}
x_{1} \\
{\left[\begin{array}{l}
x_{6} \\
x_{7} \\
x_{8}
\end{array}\right]} & {\left[\begin{array}{l}
x_{2} \\
x_{3} \\
x_{4}
\end{array}\right]} \\
x_{5}
\end{array}\right)
$$

of $\mathcal{O}(A)$ can be written $x=\sum_{i=1}^{8} x_{i} u_{i}$. It is also sometimes useful to refer to the following basis: $e_{11}=u_{1}, e_{12}=-u_{2}, f_{12}=-u_{3}, g_{12}=-u_{4}, e_{22}=u_{5}, e_{21}=u_{6}$, $f_{21}=u_{7}, g_{21}=u_{8}$. The point is that because $\left\{e_{11}, e_{12}, e_{21}, e_{22}\right\},\left\{e_{11}, f_{12}, f_{21}, e_{22}\right\}$, and $\left\{e_{11}, g_{12}, g_{21}, e_{22}\right\}$ each form a set of matrix units for $M_{2}(A)$, we obtain from them three embeddings of $M_{2}(A)$ in $\mathcal{O}(A)$.

An element $X$ of $G_{2}(A)$ is an $A$-linear isomorphism of $\mathcal{O}(A)$ and can therefore be represented by a matrix relative to the basis $\mathcal{B}$. Setting $u_{i} X=\sum_{j=1}^{8} a_{i j} u_{j}$, we identify $X$ with its $8 \times 8$ matrix $\left[a_{i j}\right]$. In this way we identify $G_{2}(A)$ with a subgroup of $G L(8, A)$. For reasons which will soon become apparent, it will also be convenient to view $X$ as a $2 \times 2$ matrix $\left[\begin{array}{ll}a(X) & b(X) \\ c(X) & d(X)\end{array}\right]$, where $a(X), b(X), c(X)$, and $d(X)$ are the $4 \times 4$ blocks of the matrix $X$. Thus,

$$
a(X)=\left[\begin{array}{llll}
a_{11} & a_{12} & a_{13} & a_{14} \\
a_{21} & a_{22} & a_{23} & a_{24} \\
a_{31} & a_{32} & a_{33} & a_{34} \\
a_{41} & a_{42} & a_{43} & a_{44}
\end{array}\right],
$$

etc.

Membership in $G_{2}(A)$. We next focus on the question of which invertible matrices $X \in G L(8, A)$ belong to $G_{2}(A)=\operatorname{Aut}(\mathcal{O}(A))$. The first thing to observe is that an invertible $X$ is in $\operatorname{Aut}(\mathcal{O}(A))$ if and only if for all $u, v \in \mathcal{O}(A)$, $(u \bar{v}) X=(u X) \overline{(v X)}$. Since $X$ is linear and every element of $\mathcal{O}(A)$ can be expressed in terms of the basis $\mathcal{B}$, we get that $X$ is in $\operatorname{Aut}(\mathcal{O}(A))$ if and only if

$$
\left(u_{i} \bar{u}_{j}\right) X=\left(u_{i} X\right) \overline{\left(u_{j} X\right)} \text { for all } 1 \leq i, j \leq 8 .
$$

Writing $\left(u_{i} X\right) \overline{\left(u_{j} X\right)}$ in terms of the basis $\mathcal{B}$, the coefficients are polynomials in the entries of $X$. Equating these with the coefficients in the expression for $\left(u_{i} \bar{u}_{j}\right) X$ gives 512 polynomial relations on the entries of $X$. Let $\mathcal{P}$ be this set of polynomials.

(A.1) An element $X \in G L(8, A)$ is in $G_{2}(A)$ if and only if the entries in $X$ satisfy the polynomials in $\mathcal{P}$.

Membership in $G_{2}(A)$ can also be expressed in terms of multiplication operators. For each $v \in \mathcal{O}(A)$, let $L_{v}: \mathcal{O}(A) \rightarrow \mathcal{O}(A)$ be the $A$-linear map $x \mapsto v x$. One easily checks that the following statement holds:

(A.2) An element $X \in G L(8, A)$ is in $G_{2}(A)$ if and only if for all $v \in \mathcal{O}(A)$, $\bar{v} X=\overline{(v X)}$ and $X^{-1} L_{v} X=L_{v X}$.

Let $P=\left[\begin{array}{ll}0 & I \\ I & 0\end{array}\right]$ where $P \in G L(8, A)$. With respect to the basis $\mathcal{B}$, we have $N(x, y)=x P y^{T}$, for all $x, y \in \mathcal{O}(A)$. If $X \in G_{2}(A)$, we have $N(x X, y X)=N(x, y)$ 
for all $x, y \in \mathcal{O}(A)$, and hence $X P X^{T}=P$. It follows that $X^{-1}=P X^{T} P$. Writing $X=\left[\begin{array}{ll}a & b \\ c & d\end{array}\right]$ in block form, we get

(A.3) If $X \in G_{2}(A)$, then $X^{-1}=\left[\begin{array}{ll}d^{T} & b^{T} \\ c^{T} & a^{T}\end{array}\right]$. Therefore, $a d^{T}+b c^{T}=1, d^{T} a+b^{T} c=$ 1 , and $a b^{T}, d c^{T}, d^{T} b, a^{T} c$ are skew-symmetric.

[We note that with the present notation, $T(x, y, z)=(x y) P z^{T}$. It follows that an invertible matrix $X$ which leaves $N(x, y)$ and $T(x, y, z)$ invariant must preserve products in $\mathcal{O}(A)$ and is easily seen to be in $G_{2}(A)$.] Note also that $P \in G_{2}(A)$, which can be shown using (A.2).

Since $1=u_{1}+u_{5}$ and any $X$ in $G_{2}(A)$ must leave the identity of $\mathcal{O}(A)$ fixed, we have $[1,0,0,0,1,0,0,0] X=[1,0,0,0,1,0,0,0]$. The same must be true of $X^{-1}$. This yields the next fact:

(A.4) If $X \in G_{2}(A)$, then the sum of the first and fifth row is [1, $\left.0,0,0,1,0,0,0\right]$, and likewise for the first and fifth column.

Defining $Q \in G L(8, A)$ by

$$
a(Q)=d(Q)=\left[\begin{array}{llll}
1 & 0 & 0 & 0 \\
0 & 0 & 0 & 0 \\
0 & 0 & 0 & 0 \\
0 & 0 & 0 & 0
\end{array}\right],
$$

and

$$
b(Q)=c(Q)=\left[\begin{array}{rrrr}
0 & 0 & 0 & 0 \\
0 & -1 & 0 & 0 \\
0 & 0 & -1 & 0 \\
0 & 0 & 0 & -1
\end{array}\right]
$$

one can check that for all $v \in \mathcal{O}(A),\left(L_{v}\right)^{T}=L_{(v Q)}$. Using (A.3) and (A.4) one sees that $Q X Q=X^{-T}$ for $X$ in $G_{2}(A)$. Combining this with (A.2) we get

(A.5) If $X \in G_{2}(A)$, then $X^{T} \in G_{2}(A)$.

We now show how these facts can be applied to find simple expressions for $a d^{T}, b c^{T}, a b^{T}, d c^{T}$, in terms of the entries of an $X=\left[a_{i j}\right]$ in $G_{2}(A)$. To this end, observe that the coefficient of $u_{1}$ in $\left(u_{i} X\right) \overline{\left(u_{j} X\right)}$ is

$$
a_{i 1} a_{j 5}+a_{i 2} a_{j 6}+a_{i 3} a_{j 7}+a_{i 4} a_{j 8}
$$

Choosing $1 \leq i \leq 4$ and $5 \leq j \leq 8$ produces the entries in $a d^{T}$. But $\left(u_{i} X\right) \overline{\left(u_{j} X\right)}=$ $\left(u_{i} \bar{u}_{j}\right) X$, so that we may compute these values by computing $u_{i} \bar{u}_{j}$ and taking the dot product with the first column of $X$. Doing so reveals that $a d^{T}=a_{11} I$. It now also follows that $b c^{T}=\left(1-a_{11}\right) I$. Applying this to $X^{T}$ and using (A.4) gives $d^{T} a=a_{11} I, b^{T} c=\left(1-a_{11}\right) I$.

Computing similarly with $1 \leq i \leq 4$ and $1 \leq j \leq 4$ gives the entries in $a b^{T}$. Applying this to $X^{-1}, X^{T}$, and $X^{-T}$, we get the following results:

(A.6) Let $X=\left[\begin{array}{ll}a & b \\ c & d\end{array}\right]=\left[a_{i j}\right]$ belong to $G_{2}(A)$. Then

(i) $a d^{T}=d^{T} a=a_{11} I$ and $b c^{T}=c^{T} b=\left(1-a_{11}\right) I$. 
(ii)

$$
\begin{aligned}
& a b^{T}=\left[\begin{array}{cccc}
0 & -a_{21} & -a_{31} & -a_{41} \\
a_{21} & 0 & -a_{81} & a_{71} \\
a_{31} & a_{81} & 0 & -a_{61} \\
a_{41} & -a_{71} & a_{61} & 0
\end{array}\right] \\
& d c^{T}=\left[\begin{array}{cccc}
0 & a_{61} & a_{71} & a_{81} \\
-a_{61} & 0 & a_{41} & -a_{31} \\
-a_{71} & -a_{41} & 0 & a_{21} \\
-a_{81} & a_{31} & -a_{21} & 0
\end{array}\right] \\
& d^{T} b=\left[\begin{array}{cccc}
0 & a_{16} & a_{17} & a_{18} \\
-a_{16} & 0 & a_{14} & -a_{13} \\
-a_{17} & -a_{14} & 0 & a_{12} \\
-a_{18} & a_{13} & -a_{12} & 0
\end{array}\right] \\
& a^{T} c=\left[\begin{array}{cccc}
0 & -a_{12} & -a_{13} & -a_{14} \\
a_{12} & 0 & -a_{18} & a_{17} \\
a_{13} & a_{18} & 0 & -a_{16} \\
a_{14} & -a_{17} & a_{16} & 0
\end{array}\right] .
\end{aligned}
$$

The case of $a_{11}$ a unit. Let $X=\left[\begin{array}{ll}a & b \\ c & d\end{array}\right]=\left[a_{i j}\right]$ be an element of $G_{2}(A)$ and suppose that $a_{11}$ is a unit in $A$. Since $a d^{T}=a_{11} I, a$ is an invertible $4 \times 4$ matrix with $a^{-1}=a_{11}^{-1} d^{T}$. From (A.4) we know that $a_{11}=a_{55}=d_{11}$, the (1,1)-entry of $d$, so that the $(1,1)$-entry of $a^{-1}$ is 1 . On the other hand, $a^{-1}=\operatorname{det}(a)^{-1} \operatorname{adj}(a)$. It follows that

If $X \in G_{2}(A)$ and $a_{11}$ is invertible, then

$$
\operatorname{det}(a)=\operatorname{det}\left[\begin{array}{ccc}
a_{22} & a_{23} & a_{24} \\
a_{32} & a_{33} & a_{34} \\
a_{42} & a_{43} & a_{44}
\end{array}\right] .
$$

Next observe that $d=a_{11} a^{-T}$. This says that $d$ is uniquely determined by the entries of $a$. Since the entries of $a$ and $d$ determine those in the first column and row of $X$ by (A.4), it follows from (A.6) that the entries of $a$ uniquely determine $b a^{T}$ and $a^{T} c$. Then $b=\left(b a^{T}\right) a^{-T}$ and $c=a^{-T}\left(a^{T} c\right)$ are also determined by the entries of $a$. We therefore have the following result:

(A.8) If $X \in G_{2}(A)$ and $a_{11}$ is invertible, then $X$ is uniquely determined by the entries of $a(X)$. 
For future reference and use it will be advantageous to note some particular relations from the set $\mathcal{P}$ of (A.1). In these we use the notation $m_{i j k \ell}$ to denote the $2 \times 2$ minor of $X$ formed using rows $i$ and $j$ and columns $k$ and $\ell$, i.e., $m_{i j k \ell}=$ $a_{i k} a_{j \ell}-a_{i \ell} a_{j k}$.

(A.9) If $X \in G_{2}(A)$, then the following relations hold:

$$
\begin{aligned}
& a_{66}=-m_{3416}+m_{3434}, \\
& a_{76}=m_{2416}-m_{2434}, \\
& a_{86}=-m_{2316}+m_{2334}, \\
& a_{67}=-m_{3417}-m_{3424}, \\
& a_{77}=m_{2417}+m_{2424}, \\
& a_{87}=-m_{2317}-m_{2324}, \\
& a_{68}=-m_{3418}+m_{3423}, \\
& a_{78}=m_{2418}-m_{2423}, \\
& a_{88}=-m_{2318}+m_{2323} .
\end{aligned}
$$

Proof. To obtain the expressions for $a_{66}, a_{67}, a_{68}$, let $i=3, j=4$ in the fundamental relation $\left(u_{i} X\right) \overline{\left(u_{j} X\right)}=\left(u_{i} \bar{u}_{j}\right) X$, and compare the coefficients of $u_{6}, u_{7}, u_{8}$. Then apply (A.4). For $a_{76}, a_{77}, a_{78}$, use $i=4, j=2$; for $a_{86}, a_{87}, a_{88}$ use $i=2, j=3$.

If for $i=6,7,8$ we define

$$
[i]= \begin{cases}34, & i=6 \\ 42, & i=7 \\ 23, & i=8\end{cases}
$$

then we may summarize these relations in the form

$$
a_{i j}=-m_{[i] 1 j}+m_{[i][j]}, \quad 6 \leq i, j \leq 8 .
$$

While we are at it, we record here some more facts about minors which we will be using:

(A.10) Plücker relations. For any matrix $X$ with entries in $A$ and any fixed positive integers $s, t$, set $\tau_{i j}=m_{s t i j}$ for all $i, j$. Then for any positive integers $i, j, k, h$,

$$
\tau_{i j} \tau_{k h}+\tau_{j k} \tau_{i h}+\tau_{k i} \tau_{j h}=0
$$

Proof. This is easily verified.

(A.11) Let $X \in G_{2}(A)$ and fix integers $s \in\{2,3,4\}$ and $t \in\{6,7,8\}$ so that $s \neq t-4$. For integers $i, j$ let $\tau_{i j}=m_{s t i j}$ as above. Then the following identities hold:

$$
\begin{aligned}
& \tau_{78}=-\tau_{12}, \tau_{68}=\tau_{13}, \tau_{67}=-\tau_{14}, \tau_{34}=\tau_{16}, \tau_{24}=-\tau_{17}, \\
& \tau_{23}=\tau_{18}, \text { and } \tau_{26}+\tau_{37}+\tau_{48}=0 .
\end{aligned}
$$

Proof. The integers $s, t$ are chosen so that $u_{s} \bar{u}_{t}=0$. Applying the fundamental identity (A.1) we have

$$
\left(u_{s} X\right) \overline{\left(u_{t} X\right)}=0 \text { in } \mathcal{O}(A) .
$$


Letting $X=\left[a_{i j}\right]$ and computing the product of

$$
u_{s} X=\left(\begin{array}{c}
a_{s 1} \\
{\left[\begin{array}{c}
a_{s 6} \\
a_{s 7} \\
a_{s 8} \\
a_{s 4}
\end{array}\right]} \\
a_{s 5}
\end{array}\right)
$$

and $\overline{\left(u_{t} X\right)}$ in terms of the $a_{i j}$ 's immediately gives $\tau_{78}=-\tau_{12}, \tau_{68}=\tau_{13}, \tau_{67}=$ $-\tau_{14}, \tau_{34}=-\tau_{56}, \tau_{24}=\tau_{57}$, and $\tau_{23}=-\tau_{58}$. Subtracting the coefficient of $e_{5}$ from the coefficient of $e_{1}$ in $\left(u_{s} X\right) \overline{\left(u_{t} X\right)}$ gives $\tau_{15}+\tau_{26}+\tau_{37}+\tau_{48}=0$. Using (A.4) one sees that $\tau_{15}=0$ and that $\tau_{5 j}=-\tau_{1 j}$ for all $j$. This completes the proof.

Scalars, diagonal elements, and the stabilizer of $e_{11}$. We now give some important facts about special elements of $G_{2}(A)$.

$$
\text { If } \alpha I \in G_{2}(A) \text { for some } \alpha \in A \text {, then } \alpha=1 \text {. }
$$

Proof. From (A.4), $a_{11}+a_{15}=1$. But $a_{11}=\alpha$ and $a_{15}=0$.

From this (or otherwise), it is easy to see that $G_{2}(A)$ has trivial center. Next we have a result on diagonal matrices in $G_{2}(A)$.

(A.13) Let $X \in G_{2}(A)$ be a diagonal matrix, say with

$$
a(X)=\left[\begin{array}{llll}
\alpha & & & 0 \\
& \beta & & \\
& & \gamma & \\
0 & & & \delta
\end{array}\right] .
$$

Then $\alpha=1$ and $\beta \gamma \delta=1$.

Proof. That $\alpha=1$ follows from (A.4) as in the previous proof. Now the second and third rows of $X$ show that $e_{12} X=\beta e_{12}$ and $f_{12} X=\gamma f_{12}$. Multiplying these equations, we get $g_{21} X=\beta \gamma g_{21}$. But $a(X) d(X)=I$, so the last row of $X$ indicates that $g_{21} X=\delta^{-1} g_{21}$, proving that $\beta \gamma \delta=1$.

(A.14) Corollary. The stabilizer of $e_{11}$ in $G_{2}(A)$ is the subgroup $S L(3, A)$.

Proof. As in Section 1, we regard $S L(3, A)$ as embedded in $G_{2}(A)$ via

$$
Y \mapsto\left[\begin{array}{cc|cc}
1 & & & 0 \\
& Y^{T} & & \\
\hline 0 & & 1 & \\
0 & & & Y^{-T}
\end{array}\right] .
$$

If $X \in \operatorname{Stab}\left(e_{11}\right)$, then since $X$ fixes the identity $1=e_{11}+e_{22}$ of $\mathcal{O}(A), X$ also fixes $e_{22}$. The same is true of $X^{-1}$ and hence the first and fifth rows and columns of $X$ 
are determined:

$$
X=\left[\begin{array}{llllllll}
1 & 0 & 0 & 0 & 0 & 0 & 0 & 0 \\
0 & & & & 0 & & & \\
0 & & * & & 0 & & * & \\
0 & & & & 0 & & & \\
0 & 0 & 0 & 0 & 1 & 0 & 0 & 0 \\
0 & & & & 0 & & & \\
0 & & * & & 0 & & * & \\
0 & & & & 0 & & &
\end{array}\right]
$$

Then $a(X)$ and $d(X)^{T}$ are inverses and by (A.6), $b(X)=c(X)=0$. So $X=\left[\begin{array}{cc}a & 0 \\ 0 & a^{-T}\end{array}\right]$ with

$$
a=\left[\begin{array}{llll}
1 & 0 & 0 & 0 \\
0 & & & \\
0 & & M & \\
0 & & &
\end{array}\right],
$$

where $M \in G L(3, A)$. Let $\delta=\operatorname{det}(M)$. Then

$$
Y=\left[\begin{array}{ccc}
\delta^{-1} & 0 & 0 \\
0 & 1 & 0 \\
0 & 0 & 1
\end{array}\right] M \in S L(3, A),
$$

and hence $X^{\prime}=\left[\begin{array}{cc}a^{\prime} & 0 \\ 0 & a^{\prime-T}\end{array}\right]$ is in $G_{2}(A)$, where

$$
a^{\prime}=\left[\begin{array}{cccc}
1 & 0 & 0 & 0 \\
0 & & & \\
0 & & Y & \\
0 & & &
\end{array}\right]
$$

Then $X\left(X^{\prime}\right)^{-1}$ is a diagonal matrix in $G_{2}(A)$ with

$$
a\left(X\left(X^{\prime}\right)^{-1}\right)=\left[\begin{array}{llll}
1 & & & \\
& \delta & & \\
& & 1 & \\
& & & 1
\end{array}\right] .
$$

By (A.13), $\delta=1$. This proves that $X \in S L(3, A)$.

The embedding $\sigma: S L(2, A) \rightarrow G_{2}(A)$ of Section 1 also gives some information we need. If $\lambda \in A$ is a unit, then $\left[\begin{array}{cc}\lambda & 0 \\ 0 & \lambda^{-1}\end{array}\right] \in E(2, A)$. In Section 1 we point out that $\sigma_{1}=\sigma$ maps $E(2, A)$ into $E(A ; A, 0)$. Now $\sigma\left(\left[\begin{array}{cc}\lambda & 0 \\ 0 & \lambda^{-1}\end{array}\right]\right)$ is a diagonal matrix with $a$-block

$$
\left[\begin{array}{cccc}
1 & 0 & 0 & 0 \\
0 & \lambda^{-2} & 0 & 0 \\
0 & 0 & \lambda & 0 \\
0 & 0 & 0 & \lambda
\end{array}\right]
$$


Note that this matrix is in the center of $S L(3, A)$ if and only if $\lambda^{3}=1$, and, furthermore, that this element is also in the other embeddings $\sigma_{2}, \sigma_{3}$ of $S L(2, A)$ in $G_{2}(A)$. From this and (A.13) we have

(A.15) Let $\lambda$ be a unit in $A$. Let $\Lambda=\operatorname{diag}\left(1, \lambda, \lambda, \lambda, 1, \lambda^{-1}, \lambda^{-1}, \lambda^{-1}\right)$. Then $\lambda^{3}=1$ if and only if

$$
\Lambda \in \operatorname{Center}(S L(3, A)) \cap \sigma_{1}(E(2, A)) \cap \sigma_{2}(E(2, A)) \cap \sigma_{3}(E(2, A)) .
$$

We close the appendix with a general fact to be used in Section 4 .

(A.16) Lemma. Let $A$ be a commutative ring, $B$ its prime subring, $n \geq 3$ an integer, and $y \in S L(n, A)$. Suppose that $E(n, B)$ centralizes $y$ in $\operatorname{PSL}(n, A)$. Then $y=\lambda I$ for some $\lambda$ in $A$.

Proof. Consider $a, b$ in $E(n, B)$. We have $[a b, y]=[a, y]^{b}[b, y]=[a, y][b, y]$, since all commutators in these equations are scalar matrices by hypothesis.

Thus, $a \rightarrow[a, y]$ is a homomorphism on $E(n, B)$ into an abelian group. But $[E(n, B), E(n, B)]=E(n, B)$ since $n \geq 3$, so the homomorphism must be trivial and $[a, y]=1$ for every $a$ in $E(n, B)$. For $i \neq j$ let $e_{i j}$ be the matrix unit in $n \times n$ matrices. Now $\left(I+e_{i j}\right) y=y\left(I+e_{i j}\right)$ for all $i \neq j$. It follows that $y$ is a scalar, as promised.

\section{REFERENCES}

[1] E. Abe, Chevalley groups over local rings, Tôhoku Math. J. 21(1969), 474-494. MR 41:3483

[2] E. Abe, Normal subgroups of Chevalley groups over commutative rings, Contemporary Mathematics, v. 83, Amer. Math. Soc., Providence, RI, 1989. MR 91a:20046

[3] E. Abe and K. Suzuki, On normal subgroups of Chevalley groups over commutative rings, Tôhoku Math. J. 28(1976), 185-198. MR 55:12828

[4] H. Bass, Algebraic K-theory, Benjamin, New York, 1968. MR 40:2736

[5] H. Bass, J. Milnor, and J.-P. Serre, Solution of the congruence subgroup problem for $S L_{n}(n \geq$ 3) and $S p_{2 n}(n \geq 2)$, Publ. Math. I.H.E.S. 33 (1967), 59-137. MR 39:5574

[6] Z. I. Borevich and N. A. Vavilov, The distribution of subgroups in the full linear group over a commutative ring, Proc. Steklov Inst. Math. 3(1985), 27-46.

[7] D. Costa and G. Keller, The $E(2, A)$ sections of $S L(2, A)$, Ann. Math. 134(1991), 159-188. MR 92f: 20047

[8] D. Costa and G. Keller, Radix redux: Normal subgroups of symplectic groups, J. Reine Angew. Math. 427(1992), 51-105. MR 93h:20053

[9] D. Costa and G. Keller, Power residue symbols and the central sections of $S L(2, A), K$-Theory 15 (1998), 33-98. CMP 99:01

[10] I. Z. Golubchik, On the general linear group over an associative ring, Uspekhi Math. Nauk. 28:3(1973), 179-180.

[11] I. Z. Golubchik, On the subgroups of the general linear group $G L_{n}(R)$ over an associative ring $R$, Russian Math. Surveys, 39:1(1984), 157-158. MR 85j:20042

[12] A. J. Hahn and O. T. O'Meara, The classical groups and $K$-theory, Springer, 1989. MR 90i: 20002

[13] N. Jacobson, Lie Algebras, Dover, 1979. MR 80k:17001

[14] G. Taddei, Normalité des groupes élémentaires dans des groupes de Chevalley sur un anneau, Contemp. Math. 55(1986), 693-708. MR 88a:20054

[15] L. N. Vaserstein, On the normal subgroups of $G L_{n}$ over a ring, Springer Lecture Notes 854(1981), 456-465. MR 83c:20058

[16] L. N. Vaserstein, On normal subgroups of Chevalley groups over commutative rings, Tôhoku Math. J. 38(1986), 219-230. MR 87k:20081 
[17] N. A. Vavilov, Structure of Chevalley groups over commutative rings, in Proc. Int. Symposium on Non-Associative Algebras and Related Topics (Hiroshima, 1990), World Scientific, 1991, 219-335. MR 92k:20090

[18] J. S. Wilson, The normal and subnormal structure of general linear groups, Proc. Cambridge Phil. Soc. 71(1972), 163-177. MR 45:398

Department of Mathematics, University of Virginia, Charlottesville, Virginia 229033199

E-mail address: dlc4v@virginia.edu

E-mail address: gek@virginia.edu 